JOURNAL OF

FUNCTION SPACES AND APPLICATIONS

Volume 7, Number 2 (2009), 121-152 (c) 2009, Scientific Horizon http://www.jfsa.net

\title{
Reiterated homogenization of nonlinear monotone operators in a general deterministic setting
}

\author{
Dag Lukkassen, Gabriel Nguetseng, Hubert Nnang \\ and Peter Wall
}

(Communicated by Björn Birnir)

2000 Mathematics Subject Classification. 35B40, 46J10.

Keywords and phrases. Reiterated homogenization, homogenization structures.

Abstract. We study reiterated homogenization of a nonlinear non-periodic elliptic differential operator in a general deterministic setting as opposed to the usual stochastic setting. Our approach proceeds from an appropriate notion of convergence termed reiterated $\Sigma$-convergence. A general deterministic homogenization theorem is proved and several concrete examples are studied under various structure hypotheses ranging from the classical periodicity hypothesis to more complicated, but realistic, structure hypotheses.

\section{Introduction}

We study the homogenization (as $0<\varepsilon \rightarrow 0$ ) of the boundary value problem

$$
-\operatorname{div} a\left(\frac{x}{\varepsilon}, \frac{x}{\varepsilon^{2}}, D u_{\varepsilon}\right)=f \text { in } \Omega, u_{\varepsilon} \in W_{0}^{1, p}(\Omega ; \mathbb{R})
$$


where $\Omega$ is a bounded open set in $\mathbb{R}_{x}^{N}$ (the $N$-dimensional numerical space of variables $\left.x=\left(x_{1}, \cdots, x_{N}\right)\right), f$ is given in $W^{-1, p^{\prime}}(\Omega ; \mathbb{R})$ with $p^{\prime}=\frac{p}{p-1}$, $1<p<\infty, D$ and div denote the usual gradient and divergence operators, respectively, in $\Omega$, and finally, $a$ is a given function $(y, z, \xi) \rightarrow a(y, z, \xi)$ from $\mathbb{R}^{N} \times \mathbb{R}^{N} \times \mathbb{R}^{N}$ to $\mathbb{R}^{N}(N \geq 1)$ with the following properties :

For any arbitrary $\xi \in \mathbb{R}^{N}$, the function $(y, z) \rightarrow a(y, z, \xi)$ possesses the Caratheodory property, i.e.,

(i) for each $z \in \mathbb{R}^{N}$, the function $y \rightarrow a(y, z, \xi)$ is measurable from $\mathbb{R}^{N}$ (with Lebesgue measure) into $\mathbb{R}^{N}$,

(ii) for almost every $y \in \mathbb{R}^{N}$, the function $z \rightarrow a(y, z, \xi)$ maps $\mathbb{R}^{N}$ continuously into $\mathbb{R}^{N}$.

$a(y, z, \omega)=\omega$ for almost all $y \in \mathbb{R}^{N}$ and for all $z \in \mathbb{R}^{N}$, where $\omega$ denotes the origin in $\mathbb{R}^{N}$.

There are four constants $c_{1}, c_{2}>0,0<\alpha_{1} \leq \min (1, p-1)$, and $\alpha_{2} \geq \max (p, 2)$ such that for almost every $y \in \mathbb{R}^{N}$ and for every $z \in \mathbb{R}^{N}$ we have

(iii) $\left|a\left(y, z, \xi_{1}\right)-a\left(y, z, \xi_{2}\right)\right| \leq$

$$
c_{1}\left(1+\left|\xi_{1}\right|+\left|\xi_{2}\right|\right)^{p-1-\alpha_{1}}\left|\xi_{1}-\xi_{2}\right|^{\alpha_{1}}
$$

(iv) $\left(a\left(y, z, \xi_{1}\right)-a\left(y, z, \xi_{2}\right)\right) \cdot\left(\xi_{1}-\xi_{2}\right) \geq$

$$
c_{2}\left(1+\left|\xi_{1}\right|+\left|\xi_{2}\right|\right)^{p-\alpha_{2}}\left|\xi_{1}-\xi_{2}\right|^{\alpha_{2}}
$$

for $\xi_{1}, \xi_{2} \in \mathbb{R}^{N}$, where the dot denotes the usual Euclidean inner product in $\mathbb{R}^{N}$, and $|\cdot|$ the associated norm.

For the sake of clearness it is well to note that the equation in (1.1) actually writes as

$$
-\operatorname{div} a^{\varepsilon}\left(\cdot, \cdot, D u_{\varepsilon}\right)=f \text { in } \Omega
$$

where $a^{\varepsilon}\left(\cdot, \cdot, D u_{\varepsilon}\right)$ stands for the function $x \rightarrow a\left(\frac{x}{\varepsilon}, \frac{x}{\varepsilon^{2}}, D u_{\varepsilon}(x)\right)$ from $\Omega$ to $\mathbb{R}^{N}$. However, since the set $Q_{\varepsilon}=\left\{\left(x, \frac{x}{\varepsilon}, \frac{x}{\varepsilon^{2}}\right): x \in \Omega\right\}$ is negligible in $\mathbb{R}_{x}^{N} \times \mathbb{R}_{y}^{N} \times \mathbb{R}_{z}^{N}$ (for Lebesgue measure $d x d y d z$ ), it is not clear that this function is well defined. Indeed, unless the function $(y, z) \rightarrow a(y, z, \xi)$ (for fixed $\xi)$ is a continuous mapping of $\mathbb{R}^{N} \times \mathbb{R}^{N}$ into $\mathbb{R}^{N}$ and $\mathbf{v}$ is taken in $\mathcal{C}(\Omega ; \mathbb{R})^{N}=\mathcal{C}(\Omega ; \mathbb{R}) \times \cdots \times \mathcal{C}(\Omega ; \mathbb{R})(N$ times $)$, it would be naive to state that $a\left(\frac{x}{\varepsilon}, \frac{x}{\varepsilon^{2}}, \mathbf{v}(x)\right)$ is the value taken by $a(y, z, \mathbf{v}(x))$ at point $\left(y=\frac{x}{\varepsilon}, z=\frac{x}{\varepsilon^{2}}\right)$. All that will be clarified in Section 2 . 
Provided the differential operator $u \rightarrow \operatorname{div} a^{\varepsilon}(\cdot, \cdot, D u), u \in W^{1, p}(\Omega ; \mathbb{R})$, is well defined and has suitable properties (see Corollary 2.1), it is a classical matter to prove an existence and uniqueness result for (1.1) (see, e.g., [16]). Thus, we have a generalized sequence $\left(u_{\varepsilon}\right)_{\varepsilon>0}$ at our disposal, and the problem is to study, under a suitable condition on $a(y, z, \xi)$ (for fixed $\xi$ ) - called a structure hypothesis, the limiting behaviour of $u_{\varepsilon}$ as $\varepsilon \rightarrow 0$. This lies within the class of so-called reiterated homogenization problems. Reiterated homogenization was introduced in [4] for linear operators. Multiscale convergence was first applied to reiterated homogenization in [8]. The reiterated homogenization of nonlinear elliptic operators was first studied in [14, 15], and latter in [16], in the usual periodic setting.

In this study we investigate the homogenization of (1.1) not under the periodicity hypothesis as in the previous references, but in a general deterministic setting including the periodicity, almost periodicity, convergence at infinity hypotheses, and others. Our approach proceeds from an appropriate notion of convergence termed reiterated $\Sigma$-convergence. A general deterministic homogenization theorem for (1.1) is established, and several examples considered in various concrete settings are presented by way of illustration. Reiterated $\Sigma$-convergence is likely to carry over to other settings. In particular, by a suitable adaptation of the approach carried out in $[2,3]$, it is possible to frame, using reiterated $\Sigma$-convergence, a reiterated homogenization theory of integral functionals in a general deterministic setting similar to that which is introduced in the present study.

The study is organized as follows. Section 2 deals with preliminary notions and results about the traces $a\left(\frac{x}{\varepsilon}, \frac{x}{\varepsilon^{2}}, \mathbf{v}(x)\right)(x \in \Omega)$ and reiterated $\Sigma$-convergence. In Section 3 we study the abstract deterministic homogenization problem for (1.1). The periodicity hypothesis stated in [14, $15,16]$ is here replaced by an abstract assumption covering a great variety of concrete structure hypotheses. Finally, Section 4 is concerned with a few concrete examples of homogenization problems for (1.1). More precisely, we consider the problem of investigating the limiting behaviour (as $\varepsilon \rightarrow 0$ ) of $u_{\varepsilon}$ (the solution of (1.1)) under various concrete structure hypotheses ranging from the classical periodicity condition to more complicated (but realistic) structure hypotheses, and we show how each of them can reduce to the abstract hypothesis in Section 3.

Except where otherwise stated, vector spaces are considered over the complex field, $\mathbb{C}$, and scalar functions assume complex values. If $X$ and $F$ denote a locally compact space and a Banach space, respectively, then $\mathcal{C}(X ; F)$ stands for the space of continuous functions of $X$ into $F$, and $\mathcal{B}(X ; F)$ stands for those functions in $\mathcal{C}(X ; F)$ that are bounded. We equip $\mathcal{B}(X ; F)$ with the supremum norm $\|u\|_{\infty}=\sup _{x \in X}\|u(x)\|$, where $\|\cdot\|$ denotes the norm in $F$. For shortness we write $\mathcal{C}(X)=\mathcal{C}(X ; \mathbb{C})$ 
and $\mathcal{B}(X)=\mathcal{B}(X ; \mathbb{C})$. Likewise the spaces $L^{p}(X ; F)$ and $L_{\text {loc }}^{p}(X ; F)$ ( $X$ provided with a positive Radon measure) are denoted by $L^{p}(X)$ and $L_{\text {loc }}^{p}(X)$, respectively, when $F=\mathbb{C}$ (we refer to $[6,7,9]$ for integration theory). Finally, it is always assumed that the numerical space $\mathbb{R}^{d}(d$ a positive integer) is equipped with Lebesgue measure $d x=d x_{1} \cdots d x_{d}$.

\section{Preliminaries}

2.1. Traces. Let $\Omega$ be a bounded open set in $\mathbb{R}_{x}^{N}$. Let $\varepsilon>0$. For $u \in L_{\text {loc }}^{1}\left(\Omega \times \mathbb{R}_{y}^{N} \times \mathbb{R}_{z}^{N}\right)=L_{\text {loc }}^{1}\left(\Omega ; L_{\text {loc }}^{1}\left(\mathbb{R}_{y}^{N} \times \mathbb{R}_{z}^{N}\right)\right)$, we set

$$
u^{\varepsilon}(x)=u\left(x, \frac{x}{\varepsilon}, \frac{x}{\varepsilon^{2}}\right) \quad(x \in \Omega)
$$

whenever the right-hand side has meaning. This is obviously the case if $u$ is continuous on $\Omega \times \mathbb{R}_{y}^{N} \times \mathbb{R}_{z}^{N}$, since the right of (2.1) is then none other than the value of $u(x, y, z)$ at $\left(y=\frac{x}{\varepsilon}, z=\frac{x}{\varepsilon^{2}}\right), x$ being given in $\Omega$. If $u$ lies in $L^{p}(\Omega ; B)(1 \leq p \leq \infty)$, where $B$ is a closed vector subspace of $\mathcal{B}\left(\mathbb{R}_{y}^{N} \times \mathbb{R}_{z}^{N}\right)$, there is no serious difficulty in verifying that the right-hand side of (2.1) still has meaning (though in a generalized sense), which determines a function $u^{\varepsilon} \in L^{p}(\Omega)$ with $\left\|u^{\varepsilon}\right\|_{L^{p}(\Omega)} \leq\|u\|_{L^{p}(\Omega ; B)}$. Our next purpose is to define $u^{\varepsilon}$ for $u \in \mathcal{C}\left(\bar{\Omega} ; L^{\infty}\left(\mathbb{R}_{y}^{N} ; \mathcal{B}\left(\mathbb{R}_{z}^{N}\right)\right)\right)$. First of all, for $\psi \in L^{\infty}\left(\mathbb{R}_{y}^{N} ; \mathcal{B}\left(\mathbb{R}_{z}^{N}\right)\right)$, put ${ }^{\varepsilon} \psi(y)=\psi\left(y, \frac{y}{\varepsilon}\right) \quad\left(y \in \mathbb{R}^{N}\right)$, which gives a function ${ }^{\varepsilon} \psi \in L^{\infty}\left(\mathbb{R}_{y}^{N}\right)$. Next, define $\psi^{\varepsilon}(x)={ }^{\varepsilon} \psi\left(\frac{x}{\varepsilon}\right)$ for $x \in \mathbb{R}^{N}$, or more explicitly

$$
\psi^{\varepsilon}(x)=\psi\left(\frac{x}{\varepsilon}, \frac{x}{\varepsilon^{2}}\right) \quad\left(x \in \mathbb{R}^{N}\right) .
$$

Clearly $\psi^{\varepsilon} \in L^{\infty}\left(\mathbb{R}^{N}\right)$ with $\left\|\psi^{\varepsilon}\right\|_{L^{\infty}\left(\mathbb{R}^{N}\right)} \leq\|\psi\|_{L^{\infty}\left(\mathbb{R}_{y}^{N} ; \mathcal{B}\left(\mathbb{R}_{z}^{N}\right)\right)}$. This being so, let $u \in \mathcal{C}(\bar{\Omega}) \otimes L^{\infty}\left(\mathbb{R}_{y}^{N} ; \mathcal{B}\left(\mathbb{R}_{z}^{N}\right)\right)$, i.e.,

$$
u=\sum_{\text {finite }} \varphi_{i} \otimes \psi_{i}, \varphi_{i} \in \mathcal{C}(\bar{\Omega}), \psi_{i} \in L^{\infty}\left(\mathbb{R}_{y}^{N} ; \mathcal{B}\left(\mathbb{R}_{z}^{N}\right)\right) .
$$

Put

$$
u^{\varepsilon}(x)=\sum_{\text {finite }} \varphi_{i}(x) \psi_{i}^{\varepsilon}(x)(x \in \Omega)
$$

where $\psi_{i}^{\varepsilon}(x)$ is defined as in (2.2). We have $u^{\varepsilon} \in L^{\infty}(\Omega)$ with $\left\|u^{\varepsilon}\right\|_{L^{\infty}(\Omega)} \leq$ $\sup _{x \in \bar{\Omega}}\|u(x)\|_{L^{\infty}\left(\mathbb{R}_{y}^{N} ; \mathcal{B}\left(\mathbb{R}_{z}^{N}\right)\right)}$ (proceed as in [17, Proposition 1.5]). Combining this with the fact that $\mathcal{C}(\bar{\Omega}) \otimes L^{\infty}\left(\mathbb{R}_{y}^{N} ; \mathcal{B}\left(\mathbb{R}_{z}^{N}\right)\right)$ is dense in $\mathcal{C}\left(\bar{\Omega} ; L^{\infty}\left(\mathbb{R}_{y}^{N} ; \mathcal{B}\left(\mathbb{R}_{z}^{N}\right)\right)\right)$, we get immediately the following 
Proposition 2.1. The operator $u \rightarrow u^{\varepsilon}$ ( $u^{\varepsilon}$ given by (2.3)-(2.4)) of $\mathcal{C}(\bar{\Omega}) \otimes L^{\infty}\left(\mathbb{R}_{y}^{N} ; \mathcal{B}\left(\mathbb{R}_{z}^{N}\right)\right)$ into $L^{\infty}(\Omega)$ extends by continuity to a continuous linear mapping, still denoted by $u \rightarrow u^{\varepsilon}$, of $\mathcal{C}\left(\bar{\Omega} ; L^{\infty}\left(\mathbb{R}_{y}^{N} ; \mathcal{B}\left(\mathbb{R}_{z}^{N}\right)\right)\right)$ into $L^{\infty}(\Omega)$ such that $\left\|u^{\varepsilon}\right\|_{L^{\infty}(\Omega)} \leq \sup _{x \in \bar{\Omega}}\|u(x)\|_{L^{\infty}\left(\mathbb{R}_{y}^{N} ; \mathcal{B}\left(\mathbb{R}_{z}^{N}\right)\right)}$ for all $u \in$ $\mathcal{C}\left(\bar{\Omega} ; L^{\infty}\left(\mathbb{R}_{y}^{N} ; \mathcal{B}\left(\mathbb{R}_{z}^{N}\right)\right)\right)$. Furthermore, if $u$ verifies $u(x, y, z) \geq 0$ for all $(x, z) \in \bar{\Omega} \times \mathbb{R}^{N}$ and for almost all $y \in \mathbb{R}^{N}$, then $u^{\varepsilon}(x) \geq 0$ for almost all $x \in \Omega$.

Thus, for $u \in \mathcal{C}\left(\bar{\Omega} ; L^{\infty}\left(\mathbb{R}_{y}^{N} ; \mathcal{B}\left(\mathbb{R}_{z}^{N}\right)\right)\right)$, the function $u^{\varepsilon}$ is defined in the sense of Proposition 2.1 and hence we are justified in still making use of the notation in (2.1).

Let us now try to give a meaning to the notation $a\left(\frac{x}{\varepsilon}, \frac{x}{\varepsilon^{2}}, D u(x)\right)(x \in \Omega)$ with $u \in W^{1, p}(\Omega, \mathbb{R})$. We will need the following lemma, which is of interest in itself.

Lemma 2.1. Let $A_{z}$ be a closed subalgebra of $\mathcal{B}\left(\mathbb{R}_{z}^{N}\right)$ and $f$ be a function of $\mathbb{R}_{y}^{N} \times \mathbb{R}_{z}^{N}$ into $\mathbb{R}$ with the following properties :

(i) Provided with the supremum norm, $A_{z}$ is separable

(ii) $A_{z}$ contains the constants

(iii) $A_{z}$ is stable under complex conjugation

(iv) For each fixed $z \in \mathbb{R}^{N}$, the function $y \rightarrow f(y, z)$ is measurable from $\mathbb{R}^{N}$ to $\mathbb{R}$

(v) For almost every $y \in \mathbb{R}^{N}$, the function $z \rightarrow f(y, z)$ of $\mathbb{R}^{N}$ into $\mathbb{R}$, denoted below by $f(y, \cdot)$, lies in $A_{z}$.

Then, the function $y \rightarrow f(y, \cdot)$ is measurable from $\mathbb{R}^{N}$ into $\mathcal{B}\left(\mathbb{R}_{z}^{N}\right)$.

Proof. It is clear that $A_{z}$ is a commutative $\mathcal{C}^{*}$-algebra with identity. Its spectrum, $\Delta\left(A_{z}\right)$, is a metrizable compact space admitting $\left\{\delta_{z}\right\}_{z \in \mathbb{R}^{N}}\left(\delta_{z}\right.$ the Dirac measure on $\mathbb{R}^{N}$ at $z$ ) as a dense subset. Furthermore, the Gelfand transformation on $A_{z}$, denoted below by $\mathcal{G}$, is an isometric isomorphism of the $\mathcal{C}^{*}$-algebra $A_{z}$ onto the $\mathcal{C}^{*}$-algebra $\mathcal{C}\left(\Delta\left(A_{z}\right)\right.$ ) (see, e.g., [13] for further details). Having made these preliminaries, let us fix an arbitrary point $s \in \Delta\left(A_{z}\right)$. Let $\left(z_{n}\right)_{n \in \mathbb{N}}$ ( $\mathbb{N}$ denotes the nonnegative integers) be a sequence in $\mathbb{R}^{N}$ such that $\delta_{z_{n}} \rightarrow s$ in $\Delta\left(A_{z}\right)$ as $n \rightarrow \infty$. Then, as $n \rightarrow \infty$, we have $\left\langle\delta_{z_{n}}, f(y, \cdot)\right\rangle \rightarrow\langle s, f(y, \cdot)\rangle=\mathcal{G}(f(y, \cdot))(s)$ for almost all $y \in \mathbb{R}^{N}$, where the brackets denote the duality pairing between $A_{z}^{\prime}$ (topological dual of $A_{z}$ ) and $A_{z}$. It follows that the function $y \rightarrow \mathcal{G}(f(y, \cdot))(s)$ is measurable from $\mathbb{R}^{N}$ to $\mathbb{R}$, since the same is true of each of the functions $y \rightarrow\left\langle\delta_{z_{n}}, f(y, \cdot)\right\rangle=f\left(y, z_{n}\right)$ ( $n$ ranging over $\mathbb{N}$ ), according to property (iv). In other words, if $\delta_{s}$ denotes the Dirac measure on $\Delta\left(A_{z}\right)$ at $s$, and $g$ denotes the function $y \rightarrow \mathcal{G}(f(y, \cdot))$ from $\mathbb{R}^{N}$ to $\mathcal{C}\left(\Delta\left(A_{z}\right)\right)$, then the 
function $y \rightarrow\left\langle\delta_{s}, g(y)\right\rangle$ is measurable from $\mathbb{R}^{N}$ into $\mathbb{R}$ and that for any arbitrary $s \in \Delta\left(A_{z}\right)$. Therefore, given any arbitrary Radon measure $\rho$ on $\Delta\left(A_{z}\right)$ with finite support, i.e., $\rho$ of the form

$$
\rho=\sum_{\text {finite }} c_{i} \delta_{s_{i}}\left(c_{i} \in \mathbb{C} \text { and } s_{i} \in \Delta\left(A_{z}\right)\right),
$$

the function $y \rightarrow\langle\rho, g(y)\rangle$ is measurable from $\mathbb{R}^{N}$ into $\mathbb{C}$, the brackets denoting this time the duality pairing between $\mathcal{M}\left(\Delta\left(A_{z}\right)\right)=\mathcal{C}^{\prime}\left(\Delta\left(A_{z}\right)\right)$ (space of complex Radon measures on $\left.\Delta\left(A_{z}\right)\right)$ and $\mathcal{C}\left(\Delta\left(A_{z}\right)\right)$. With this in mind, fix freely a Radon measure $\eta$ on $\Delta\left(A_{z}\right)$. Note that $\eta$ is bounded, since $\Delta\left(A_{z}\right)$ is compact. Thus, we may assume without loss of generality that $\eta$ lies in the closed unit ball $B^{\prime} \subset \mathcal{M}\left(\Delta\left(A_{z}\right)\right)$. But $B^{\prime}$ with the relative weak $*$ topology on $\mathcal{M}\left(\Delta\left(A_{z}\right)\right)$ is a metrizabble compact space (the compacity is classical, the metrizability follows by property (i)). Hence, recalling a classical result (see, e.g., [6, Chap.III, p.71, Corol.1]), we may consider a sequence

$$
\left(\rho_{n}\right)_{n \in \mathbb{N}}, \rho_{n} \in B^{\prime}, \rho_{n} \text { with finite support }
$$

such that $\rho_{n} \rightarrow \eta$ in $\mathcal{M}\left(\Delta\left(A_{z}\right)\right.$ ) (with the weak $*$ topology) as $n \rightarrow \infty$. Consequently, a.e. in $y \in \mathbb{R}^{N},\left\langle\rho_{n}, g(y)\right\rangle \rightarrow\langle\eta, g(y)\rangle$ as $n \rightarrow \infty$. We deduce by a classical argument that the function $y \rightarrow\langle\eta, g(y)\rangle$ is measurable from $\mathbb{R}^{N}$ into $\mathbb{C}$. Therefore, in view of the arbitrariness of $\eta$, the lemma follows by [6, Chap.IV; p.182, Corol.2, and p.174, Thm 1].

At the present time, we assume that

$$
a_{i}(y, \cdot, \xi) \in A_{z} \quad(1 \leq i \leq N) \text { for all } \xi \in \mathbb{R}^{N} \text { and for almost all } y \in \mathbb{R}^{N}
$$

where $a_{i}(y, \cdot, \xi)$ stands for the function $z \rightarrow a_{i}(y, z, \xi)$ of $\mathbb{R}^{N}$ into $\mathbb{R}$, and $A_{z}$ is a closed subalgebra of $\mathcal{B}\left(\mathbb{R}_{z}^{N}\right)$ with the properties (i)-(iii) of Lemma 2.1. We will see that condition (2.5) is fulfilled in practice (see the forthcoming remark).

Remark 2.1. By combining [part (ii) of] (1.2) with (1.3) and [part (iii) of] (1.4), we have immediately $a_{i}(y, \cdot, \xi) \in \mathcal{B}\left(\mathbb{R}_{z}^{N}\right)(1 \leq i \leq N)$ for all $\xi \in \mathbb{R}^{N}$ and for almost all $y \in \mathbb{R}^{N}$. Unfortunately Lemma 2.1 does not apply because the space $\mathcal{B}\left(\mathbb{R}_{z}^{N}\right)$ is not separable. We will see that a condition such as (2.5) is nevertheless fulfilled in practice as a consequence of the concrete structure hypothesis on $a_{i}(y, z, \xi)$ (for fixed $\xi$ ); see Section 4 . 
Now, recalling (1.2)-(1.4), we see that Lemma 2.1 applies with $f(y, z)=$ $a_{i}(y, z, \xi)$, where $i$ and $\xi$ are freely fixed. Hence

$$
a_{i}(\cdot, \cdot, \xi) \in L^{\infty}\left(\mathbb{R}_{y}^{N} ; \mathcal{B}\left(\mathbb{R}_{z}^{N}\right)\right)(1 \leq i \leq N)
$$

for any fixed $\xi \in \mathbb{R}^{N}$, where $a_{i}(\cdot, \cdot, \xi)$ denotes the function $(y, z) \rightarrow$ $a_{i}(y, z, \xi)$ of $\mathbb{R}^{N} \times \mathbb{R}^{N}$ into $\mathbb{R}$. With this in mind, let $\mathbf{v} \in \mathcal{C}_{\mathbb{R}}(\bar{\Omega})^{N}=\mathcal{C}_{\mathbb{R}}(\bar{\Omega}) \times$ $\cdots \times \mathcal{C}_{\mathbb{R}}(\bar{\Omega})(N$ times $)$, where $\mathcal{C}_{\mathbb{R}}(\bar{\Omega})=\mathcal{C}(\bar{\Omega} ; \mathbb{R})$. The function $(x, y, z) \rightarrow$ $a_{i}(y, z, \mathbf{v}(x))$ of $\bar{\Omega} \times \mathbb{R}_{y}^{N} \times \mathbb{R}_{z}^{N}$ into $\mathbb{R}$ lies in $\mathcal{C}\left(\bar{\Omega} ; L^{\infty}\left(\mathbb{R}_{y}^{N} ; \mathcal{B}\left(\mathbb{R}_{z}^{N}\right)\right)\right.$ ) (the verification is an easy matter). Hence, according to Proposition 2.1, we can define the function $x \rightarrow a_{i}\left(\frac{x}{\varepsilon}, \frac{x}{\varepsilon^{2}}, \mathbf{v}(x)\right)$ from $\Omega$ into $\mathbb{R}$, which belongs to $L_{\mathbb{R}}^{\infty}(\Omega)=L^{\infty}(\Omega ; \mathbb{R})$ and is denoted below by $a_{i}^{\varepsilon}(\cdot, \cdot, \mathbf{v})$.

Proposition 2.2. Let $1<p<\infty$. Suppose (2.5) holds. For each fixed integer $1 \leq i \leq N$, the transformation $\mathbf{v} \rightarrow a_{i}^{\varepsilon}(\cdot, \cdot, \mathbf{v})$ of $\mathcal{C}_{\mathbb{R}}(\bar{\Omega})^{N}$ into $L_{\mathbb{R}}^{\infty}(\Omega)$ extends by continuity to a continuous mapping, still denoted by $\mathbf{v} \rightarrow a_{i}^{\varepsilon}(\cdot, \cdot, \mathbf{v})$, of $L_{\mathbb{R}}^{p}(\Omega)^{N}$ into $L_{\mathbb{R}}^{p^{\prime}}(\Omega) \quad\left(p^{\prime}=\frac{p}{p-1}\right)$. Furthermore, on letting

$$
a^{\varepsilon}(\cdot, \cdot, \mathbf{v})=\left(a_{i}^{\varepsilon}(\cdot, \cdot, \mathbf{v})\right)_{1 \leq i \leq N} \text { for } \mathbf{v} \in L_{\mathbb{R}}^{p}(\Omega)^{N}
$$

we have

$$
\begin{aligned}
\left\|a^{\varepsilon}(\cdot, \cdot, \mathbf{v})-a^{\varepsilon}(\cdot, \cdot, \mathbf{w})\right\|_{L^{p^{\prime}}(\Omega)^{N}} \leq \\
\quad c_{1}\|1+|\mathbf{v}|+|\mathbf{w}|\|_{L^{p}(\Omega)}^{p-1-\alpha_{1}}\|\mathbf{v}-\mathbf{w}\|_{L^{p}(\Omega)^{N}}^{\alpha_{1}}
\end{aligned}
$$

and

$$
\begin{aligned}
& \left(a^{\varepsilon}(\cdot, \cdot, \mathbf{v})-a^{\varepsilon}(\cdot, \cdot, \mathbf{w})\right) \cdot(\mathbf{v}-\mathbf{w}) \geq \\
& \quad c_{2}(1+|\mathbf{v}|+|\mathbf{w}|)^{p-\alpha_{2}}|\mathbf{v}-\mathbf{w}|^{\alpha_{2}} \text { a.e. in } \Omega
\end{aligned}
$$

for all $\mathbf{v}, \mathbf{w} \in L_{\mathbb{R}}^{p}(\Omega)^{N}$.

Proof. This follows in view of the proof of [19, Proposition 2.1].

As a direct consequence of this, we have the following

Corollary 2.1. Let the hypotheses of Proposition 2.2 be satisfied. For $u \in W^{1, p}(\Omega ; \mathbb{R})$, let $a^{\varepsilon}(\cdot, \cdot, D u)=\left(a_{i}^{\varepsilon}(\cdot, \cdot, D u)\right)_{1 \leq i \leq N}$ be defined as in Proposition 2.2, which gives a mapping $u \rightarrow a^{\varepsilon}(\cdot, \cdot, D u)$ of $W^{1, p}(\Omega ; \mathbb{R})$ into $L^{p^{\prime}}(\Omega)^{N}$. We have

$$
\begin{aligned}
&\left\|a^{\varepsilon}(\cdot, \cdot, D u)-a^{\varepsilon}(\cdot, \cdot, D v)\right\|_{L^{p^{\prime}}(\Omega)^{N}} \leq \\
& c_{1}\|1+|D u|+|D v|\|_{L^{p}(\Omega)}^{p-1-\alpha_{1}}\|D u-D v\|_{L^{p}(\Omega)^{N}}^{\alpha_{1}}
\end{aligned}
$$

and

$$
\begin{aligned}
& \left(a^{\varepsilon}(\cdot, \cdot, D u)-a^{\varepsilon}(\cdot, \cdot, D v)\right) \cdot(D u-D v) \geq \\
& c_{2}(1+|D u|+|D v|)^{p-\alpha_{2}}|D u-D v|^{\alpha_{2}} \text { a.e. in } \Omega
\end{aligned}
$$


for all $u, v \in W^{1, p}(\Omega ; \mathbb{R})$.

Remark 2.2. It is sometimes convenient to denote the function $a_{i}^{\varepsilon}(\cdot, \cdot, D u)$ (with $\left.u \in W^{1, p}(\Omega ; \mathbb{R})\right)$ by $x \rightarrow a_{i}^{\varepsilon}\left(\frac{x}{\varepsilon}, \frac{x}{\varepsilon^{2}}, D u(x)\right)$. However, though entirely justified above, this is merely a formal notation.

2.2. Reiterated $\Sigma$-convergence. Let us first state some fundamentals of homogenization structures beyond the classical two-scale setting.

Let $d$ be a positive integer. Let $\mathcal{H}=\left(H_{\varepsilon}\right)_{\varepsilon>0}$ be an action of $\mathbb{R}_{+}^{*}$ (the multiplicative group of positive real numbers) on the numerical space $\mathbb{R}^{d}$, i.e., $\mathcal{H}$ is a family, indexed by $\mathbb{R}_{+}^{*}$, of permutations $H_{\varepsilon}$ of $\mathbb{R}^{d}$ such that

(i) $H_{\varepsilon} \circ H_{\varepsilon^{\prime}}=H_{\varepsilon \varepsilon^{\prime}}$ for all $\varepsilon, \varepsilon^{\prime}>0$

(ii) $H_{\varepsilon=1}=I d_{\mathbb{R}^{d}}$,

where $\circ$ and $I d_{\mathbb{R}^{d}}$ denote usual composition and the identical mapping, respectively, of $\mathbb{R}^{d}$. We assume further that :

$(H)_{1}$ Each $H_{\varepsilon}$ maps continuously $\mathbb{R}^{d}$ into itself.

$(H)_{2} \lim _{\varepsilon \rightarrow 0}\left|H_{\varepsilon}(x)\right|=+\infty$ for any $x \in \mathbb{R}^{d}$ with $x \neq \omega$, where $|\cdot|$ and $\omega$ denote the Euclidean norm and the origin in $\mathbb{R}^{d}$, respectively.

$(H)_{3}$ The Lebesgue measure $\lambda$ on $\mathbb{R}^{d}$ is quasi-invariant under $\mathcal{H}$, i.e., to each $\varepsilon>0$ there is attached some $\gamma(\varepsilon)>0$ such that $H_{\varepsilon}(\lambda)=$ $\gamma(\varepsilon) \lambda$.

Remark 2.3. In view of $(H)_{1}$, the mapping $H_{\varepsilon}$ is a homeomorphism of $\mathbb{R}^{d}$ onto itself and therefore the image measure $H_{\varepsilon}(\lambda)$ is well defined (see, e.g., [7]). We recall that $H_{\varepsilon}(\lambda)$ is the Radon measure on $\mathbb{R}^{d}$ given by $\left\langle H_{\varepsilon}(\lambda), \varphi\right\rangle=\int \varphi\left(H_{\varepsilon}(x)\right) d x$ for $\varphi \in \mathcal{K}\left(\mathbb{R}^{d}\right)$ (space of compactly supported continuous complex functions on $\left.\mathbb{R}^{d}\right)$, or equivalently by $H_{\varepsilon}(\lambda)(B)=$ $\lambda\left(H_{\varepsilon}^{-1}(B)\right)$ for any bounded Borel set $B \subset \mathbb{R}^{d}$. In view of $(H)_{3}$, the transformation $u \rightarrow u \circ H_{\varepsilon}$ (usual composition) maps $L_{l o c}^{p}\left(\mathbb{R}^{d}\right)$ (resp. $\left.L^{p}\left(\mathbb{R}^{d}\right)\right)$ into itself, $1 \leq p \leq \infty$.

We denote by $\Pi^{\infty}\left(\mathbb{R}^{d}, \mathcal{H}\right)$, or simply $\Pi^{\infty}$ when there is no danger of confusion, the space of those $u \in \mathcal{B}\left(\mathbb{R}^{d}\right)$ for which a complex number $M(u)$ exists such that $u \circ H_{\varepsilon} \rightarrow M(u)$ in $L^{\infty}\left(\mathbb{R}^{d}\right)$-weak $*$ as $\varepsilon \rightarrow 0$. $\Pi^{\infty}$ is a closed vector subspace of $\mathcal{B}\left(\mathbb{R}^{d}\right), \Pi^{\infty}$ contains the constants, $\Pi^{\infty}$ is stable under complex conjugation. Furthermore, the mapping $u \rightarrow M(u)$ of $\Pi^{\infty}$ into $\mathbb{C}$ is a positive linear form on $\Pi^{\infty}$ attaining the value 1 on the constant function 1 . Such a linear form is necessarily continuous and of norm exactly one. We call $M$ the mean value on $\mathbb{R}^{d}$ for $\mathcal{H}$.

We are now in a position to introduce the notion of a homogenization structure in the present general setting. We begin by setting an underlying 
notion. By a structural representation on $\mathbb{R}^{d}$ for the action $\mathcal{H}$ is meant any set $\Gamma \subset \mathcal{B}\left(\mathbb{R}^{d}\right)$ with the properties :

(HS1) $\Gamma$ is a group under multiplication

(HS2) $\Gamma$ is countable

(HS3) $\Gamma$ is stable under complex conjugation

(HS4) $\Gamma \subset \Pi^{\infty}$.

Next, in the collection of all structural representations on $\mathbb{R}^{d}$ for $\mathcal{H}$, we consider the equivalence relation $\sim$ defined as : $\Gamma \sim \Gamma^{\prime}$ if and only if $C L S(\Gamma)=C L S\left(\Gamma^{\prime}\right)$, where $C L S(\Gamma)$ denotes the closed vector subspace of $\mathcal{B}\left(\mathbb{R}^{d}\right)$ spanned by $\Gamma$. By an $H$-structure on $\mathbb{R}^{d}$ for $\mathcal{H}$ ( $H$ stands for homogenization) we shall understand any equivalence class modulo $\sim$.

The notion of an $H$-structure is intimately connected with that of an $H$-algebra. Specifically, let $\Sigma$ be an $H$-structure on $\mathbb{R}^{d}$ for $\mathcal{H}$. Let $A=C L S(\Gamma)$, where $\Gamma$ is any equivalence class representative of $\Sigma$ (such a $\Gamma$ is termed a representation of $\Sigma$ ). The space $A$ is a so-called $H$-algebra on $\mathbb{R}^{d}$ for $\mathcal{H}$, i.e., a closed subalgebra of $\mathcal{B}\left(\mathbb{R}^{d}\right)$ with the features :

(HA1) $A$ with the supremum norm is separable

(HA2) $A$ contains the constants

(HA3) $A$ is stable under complex conjugation

(HA4) $A \subset \Pi^{\infty}$.

Furthermore, $A$ depends only on $\Sigma$ and not on the chosen representation $\Gamma$ of $\Sigma$; so that we may set $A=\mathcal{J}(\Sigma)$ (the image of $\Sigma$ ), which yields a mapping $\Sigma \rightarrow \mathcal{J}(\Sigma)$ that carries the collection of all $H$-structures (for $\mathcal{H}$ ) bijectively over the collection of all $H$-algebras (for $\mathcal{H}$ ) (see [18, Theorem $3.1])$.

It is an easy matter to see that the theory of $H$-structures developed earlier in the particular setting of [18] carries over to the present general setting. Thus, basic notions such as the partial derivatives on $\Delta(A)$ ( $A$ a given $H$-algebra on $\mathbb{R}^{d}$ for $\mathcal{H}$ ), the Sobolev spaces $W^{1, p}(\Delta(A))$, the $\Sigma$ convergence, etc., remain valid and hence are not worth repeating here. We refer the reader to $[18,19]$ for further details.

In the present work we are concerned with three specific actions of $\mathbb{R}_{+}^{*}$ : the action $\mathcal{H}=\left(H_{\varepsilon}\right)_{\varepsilon>0}$ on $\mathbb{R}^{N}$ (integer $N \geq 1$ ) given by $H_{\varepsilon}(x)=\frac{x}{\varepsilon}$ $\left(x \in \mathbb{R}^{N}\right)$, the action $\mathcal{H}^{\prime}=\left(H_{\varepsilon}^{\prime}\right)_{\varepsilon>0}$ on $\mathbb{R}^{N}$ given by $H_{\varepsilon}^{\prime}(x)=\frac{x}{\varepsilon^{2}}\left(x \in \mathbb{R}^{N}\right)^{\varepsilon}$, and their product $\mathcal{H}^{*}=\mathcal{H} \times \mathcal{H}^{\prime}$, which is precisely the action of $\mathbb{R}_{+}^{*}$ on $\mathbb{R}^{2 N}=\mathbb{R}^{N} \times \mathbb{R}^{N}$ defined by $\mathcal{H}^{*}=\left(H_{\varepsilon}^{*}\right)_{\varepsilon>0}, H_{\varepsilon}^{*}=H_{\varepsilon} \times H_{\varepsilon}^{\prime}$ (direct product), i.e.,

$$
H_{\varepsilon}^{*}\left(x, x^{\prime}\right)=\left(\frac{x}{\varepsilon}, \frac{x^{\prime}}{\varepsilon^{2}}\right) \text { for }\left(x, x^{\prime}\right) \in \mathbb{R}^{N} \times \mathbb{R}^{N} .
$$


Each of these three actions satisfies properties $(H)_{1}-(H)_{3}$, mutatis mutandis.

Now, let $\Sigma_{y}$ be an $H$-structure of class $\mathcal{C}^{\infty}$ on $\mathbb{R}_{y}^{N}$ for $\mathcal{H}$, and $\Sigma_{z}$ be an $H$-structure of class $\mathcal{C}^{\infty}$ on $\mathbb{R}_{z}^{N}$ for $\mathcal{H}^{\prime}$. Their product $\Sigma=\Sigma_{y} \times \Sigma_{z}$ is defined exactly as in [18, Definition 3.4], and is an $H$-structure of class $\mathcal{C}^{\infty}$ on $\mathbb{R}^{N} \times \mathbb{R}^{N}$ for the product action $\mathcal{H}^{*}$. It is an elementary exercise to verify that Proposition 3.2, Theorem 3.2 and Corollaries 3.1-3.2 of [18] carry over mutatis mutandis to the present context. We will put $A_{y}=\mathcal{J}\left(\Sigma_{y}\right)$ (image of $\left.\Sigma_{y}\right), A_{z}=\mathcal{J}\left(\Sigma_{z}\right)$ and $A=\mathcal{J}(\Sigma)$, and use the same letter, $\mathcal{G}$, to denote the Gelfand transformation on $A_{y}, A_{z}$ and $A$, as well. Points in $\Delta\left(A_{y}\right)$ (resp. $\left.\Delta\left(A_{z}\right)\right)$ are denoted by $s$ (resp. $r$ ). The compact space $\Delta\left(A_{y}\right)$ (resp. $\left.\Delta\left(A_{z}\right)\right)$ is equipped with the so-called $M$-measure, $\beta_{y}$ (resp. $\beta_{z}$ ) for $A_{y}$ (resp. $\left.A_{z}\right)$. It is fundamental to recall that $\Delta(A)=\Delta\left(A_{y}\right) \times \Delta\left(A_{z}\right)$ (cartesian product) and further the $M$-measure for $A$, with which $\Delta(A)$ is equipped, is precisely the product measure $\beta=\beta_{y} \otimes \beta_{z}$ (see [18]).

Before we can introduce the concept of reiterated $\Sigma$-convergence, we require one further notion.

Definition 2.1. The $H$-structure $\Sigma=\Sigma_{y} \times \Sigma_{z}$ is called a reiteration $H$-structure if for each $\psi \in A=\mathcal{J}(\Sigma)$ (image of $\Sigma$ ), we have $\psi^{\varepsilon} \rightarrow M(\psi)$ in $L^{\infty}\left(\mathbb{R}_{x}^{N}\right)$-weak $*$ as $\varepsilon \rightarrow 0$, where $\psi^{\varepsilon}$ is defined (in an obvious manner) in (2.2), and $M$ is the mean value on $\mathbb{R}^{2 N}=\mathbb{R}^{N} \times \mathbb{R}^{N}$ for the product action $\mathcal{H}^{*}$.

Remark 2.4. According to (HA4), for each $\psi \in A=\mathcal{J}(\Sigma)$ we have $\psi \circ H_{\varepsilon}^{*} \rightarrow M(\psi)$ in $L^{\infty}\left(\mathbb{R}_{x}^{N} \times \mathbb{R}_{x^{\prime}}^{N}\right)$-weak $*$, which is very different from the convergence property in the above definition.

We give below a few examples of reiteration $H$-structures.

Example 2.1. Let $\Gamma=\left\{\gamma_{k}: k \in \mathbb{Z}^{N}\right\}$ ( $\mathbb{Z}$ denotes the integers), where for each $k \in \mathbb{R}^{N}$, we write $\gamma_{k}$ for the usual exponential function on $\mathbb{R}^{N}$, i.e., $\gamma_{k}(y)=\exp (2 i \pi k \cdot y)\left(y \in \mathbb{R}^{N}\right)$. The set $\Gamma$ is a structural representation on $\mathbb{R}^{N}$ for $\mathcal{H}$ and $\mathcal{H}^{\prime}$, as well. We define $\Sigma_{\mathbb{Z}^{N}}$ (resp. $\Sigma_{\mathbb{Z}^{N}}^{\prime}$ ) to be the unique $H$-structure on $\mathbb{R}^{N}$ for $\mathcal{H}$ (resp. $\mathcal{H}^{\prime}$ ) of which $\Gamma$ is one representation. $\Sigma_{\mathbb{Z}^{N}}$ is referred to as the periodic $H$-structure on $\mathbb{R}^{N}$ represented by $\mathbb{R}^{N}$ (see $\left[18\right.$, Example 3.2]). We have $\mathcal{J}\left(\Sigma_{\mathbb{Z}^{N}}\right)=\mathcal{J}\left(\Sigma_{\mathbb{Z}^{N}}^{\prime}\right)=\mathcal{P}\left(\mathbb{R}^{N}\right)$, where $\mathcal{P}\left(\mathbb{R}^{N}\right)$ is the space of functions $u \in \mathcal{C}\left(\mathbb{R}^{N}\right)$ such that $u(y+k)=u(y)$ for all $y \in \mathbb{R}^{N}$ and all $k \in \mathbb{Z}^{N}$ (such functions are said to be $\mathbb{Z}^{N}$-periodic). Hence $\Sigma_{\mathbb{Z}^{N}}^{\prime}=\Sigma_{\mathbb{Z}^{N}}$. Finally, we consider the product $H$-structure $\Sigma=\Sigma_{\mathbb{Z}^{N}} \times \Sigma_{\mathbb{Z}^{N}}$ on $\mathbb{R}_{y}^{N} \times \mathbb{R}_{z}^{N}$ for $\mathcal{H}^{*}$. It can be proved that $\Sigma$ is a reiteration $H$-structure (the verification is left to the reader). 
Example 2.2. Let $\Gamma_{y}=\left\{\gamma_{k}: k \in \mathcal{R}_{y}\right\}$ and $\Gamma_{z}=\left\{\gamma_{k}: k \in \mathcal{R}_{z}\right\}$, where $\mathcal{R}_{y}$ and $\mathcal{R}_{z}$ are countable subgroups of $\mathbb{R}^{N}$. The set $\Gamma_{y}$ (resp. $\Gamma_{z}$ ) is a structural representation on $\mathbb{R}^{N}$ for $\mathcal{H}$ (resp. $\mathcal{H}^{\prime}$ ). We define $\Sigma_{\mathcal{R}_{y}}$ (resp. $\Sigma_{\mathcal{R}_{z}}$ ) to be the unique $H$-structure on $\mathbb{R}^{N}$ for $\mathcal{H}$ (resp. $\mathcal{H}^{\prime}$ ) of which $\Gamma_{y}$ (resp. $\Gamma_{z}$ ) is one representation. $\Sigma_{\mathcal{R}_{y}}$ (resp. $\Sigma_{\mathcal{R}_{z}}$ ) is referred to as the almost periodic $H$-structure on $\mathbb{R}^{N}$ represented by $\mathcal{R}_{y}$ (resp. $\mathcal{R}_{z}$ ), see [18, Example 3.3]. According to [18, Example 3.6], the product $\Sigma=\Sigma_{\mathcal{R}_{y}} \times \Sigma_{\mathcal{R}_{z}}$, which is an $H$-structure on $\mathbb{R}^{N} \times \mathbb{R}^{N}$ for $\mathcal{H}^{*}$, is precisely the almost periodic $H$-structure on $\mathbb{R}^{2 N}=\mathbb{R}^{N} \times \mathbb{R}^{N}$ represented by the countable subgroup $\mathcal{R}=\mathcal{R}_{y} \times \mathcal{R}_{z}$ of $\mathbb{R}^{2 N}$. Thus, $\Sigma=\Sigma_{\mathcal{R}}$, and further there is no serious difficulty in verifying that $\Sigma$ is a reiteration $H$-stucture.

Example 2.3. Let $\Sigma_{\infty}$ be the so-called $H$-structure of the convergence at infinity on $\mathbb{R}^{N}\left[18\right.$, Example 3.4]. This is an $H$-structure on $\mathbb{R}^{N}$ for $\mathcal{H}$ and $\mathcal{H}^{\prime}$, as well. The product $\Sigma=\Sigma_{\infty} \times \Sigma_{\infty}$ is an $H$-structure on $\mathbb{R}^{N} \times \mathbb{R}^{N}$ for the product action $\mathcal{H}^{*}$. In view of [18, Proposition 3.2], we have $\mathcal{J}(\Sigma)=\mathcal{B}_{\infty}\left(\mathbb{R}_{y}^{N} ; \mathcal{B}_{\infty}\left(\mathbb{R}_{z}^{N}\right)\right)$, from which one can easily check that $\Sigma$ is a reiteration $H$-structure.

Example 2.4. Let $\Sigma=\Sigma_{y} \times \Sigma_{\infty}$, where $\Sigma_{z}=\Sigma_{\infty}$ is as in Example 2.3 and $\Sigma_{y}$ is any $H$-structure of class $\mathcal{C}^{\infty}$ on $\mathbb{R}^{N}$ for $\mathcal{H}$. $\Sigma$ is an $H$-structure on $\mathbb{R}^{N} \times \mathbb{R}^{N}$ for $\mathcal{H}^{*}$. Furthermore, $\mathcal{J}(\Sigma)=\mathcal{B}_{\infty}\left(\mathbb{R}_{z}^{N} ; A_{y}\right)$ with $A_{y}=\mathcal{J}\left(\Sigma_{y}\right)$ (proceed as in [18, Example 3.7]), from which one can easily deduce that $\Sigma$ is a reiteration $H$-structure.

Example 2.5. Let $\Sigma=\Sigma_{\mathcal{R}_{y}} \times \Sigma_{\infty, \mathcal{R}_{z}}$ where $\mathcal{R}_{y}, \mathcal{R}_{z}$ and $\Sigma_{\mathcal{R}_{y}}$ are as in Example 2.2, and $\Sigma_{\infty, \mathcal{R}_{z}}$ is the $H$-structure on $\mathbb{R}^{N}$ for $\mathcal{H}^{\prime}$ defined in [18, Example 3.5]. This is an $H$-structure on $\mathbb{R}^{N} \times \mathbb{R}^{N}$ for $\mathcal{H}^{*}$. It can be shown that $\Sigma$ is a reiteration $H$-structure.

Example 2.6. Let $\Sigma=\Sigma_{\infty, \mathcal{R}_{y}} \times \Sigma_{\infty, \mathcal{R}_{z}}$ where $\mathcal{R}_{y}$ and $\mathcal{R}_{z}$ are as above. This is clearly an $H$-structure on $\mathbb{R}^{N} \times \mathbb{R}^{N}$ for $\mathcal{H}^{*}$, and there is no serious difficulty in verifying that $\Sigma$ is a reiteration $H$-stucture.

Returning now to the preceding general framework, we assume from now that the $H$-structure $\Sigma=\Sigma_{y} \times \Sigma_{z}$ is a reiteration $H$-structure. The letter $\Omega$ throughout will denote a bounded open set in $\mathbb{R}_{x}^{N}$. Here is a fundamental result.

Proposition 2.3. As $\varepsilon \rightarrow 0$, we have $u^{\varepsilon} \rightarrow \widetilde{u}$ in $L^{\infty}(\Omega)$-weak $*$ for $u \in \mathcal{C}(\bar{\Omega} ; A)$ and $u^{\varepsilon} \rightarrow \widetilde{u}$ in $L^{p}(\Omega)$-weak for $u \in L^{p}(\Omega ; A) \quad(1 \leq p<\infty)$, where $u^{\varepsilon}$ is defined in (2.1) and $\widetilde{u}$ denotes the complex function on $\Omega$ given by $\widetilde{u}(x)=M(u(x))(x \in \Omega), M$ as in Definition 2.1. 
Proof. Starting from the convergence property in Definition 2.1, we see immediately that the proposition follows by the density of $\mathcal{C}(\bar{\Omega}) \otimes A$ in $\mathcal{C}(\bar{\Omega} ; A)$ and by that of $\mathcal{C}(\bar{\Omega} ; A)$ in $L^{p}(\Omega ; A)$.

We are now ready to introduce the concepts of reiterated weak and strong $\Sigma$-convergence. The letter $E$ throughout will denote a family of positive real numbers admitting 0 as an accumulation point. For example $E=\mathbb{R}_{+}^{*}$. Attention is drawn to the especial case where $E=\left(\varepsilon_{n}\right)$ (integers $n \geq 0$ ) with $\varepsilon_{n}>0$ and $\varepsilon_{n} \rightarrow 0$ as $n \rightarrow \infty ; E$ is then referred to as a fundamental sequence.

Definition 2.2. A sequence $\left(u_{\varepsilon}\right)_{\varepsilon \in E} \subset L^{p}(\Omega) \quad(1 \leq p<\infty)$ is said to : (i) weakly $\Sigma$-converge reiteratively in $L^{p}(\Omega)$ to some $u_{0} \in L^{p}(\Omega \times \Delta(A))$ if as $E \ni \varepsilon \rightarrow 0$, we have

$$
\int_{\Omega} u_{\varepsilon} v^{\varepsilon} d x \rightarrow \iint_{\Omega \times \Delta(A)} u_{0} \widehat{v} d x d \beta
$$

for every $v \in L^{p^{\prime}}(\Omega ; A)\left(\frac{1}{p^{\prime}}=1-\frac{1}{p}\right)$, where $v^{\varepsilon}$ is defined as in (2.1), and $\widehat{v}=\mathcal{G} \circ v$ (i.e., $\widehat{v}$ denotes the function in $L^{p^{\prime}}(\Omega ; \mathcal{C}(\Delta(A)))$ given by $\widehat{v}(x)=\mathcal{G}(v(x)), x \in \Omega)$;

(ii) strongly $\Sigma$-converge reiteratively in $L^{p}(\Omega)$ to some $u_{0} \in L^{p}(\Omega \times \Delta(A))$ if the following condition is fulfilled :

$$
\text { Given } \eta>0 \text { and } v \in L^{p}(\Omega ; A) \text { with }\left\|u_{0}-\widehat{v}\right\|_{L^{p}(\Omega \times \Delta(A))} \leq \frac{\eta}{2} \text {, there }
$$
is some $\alpha>0$ such that $\left\|u_{\varepsilon}-v^{\varepsilon}\right\|_{L^{p}(\Omega)} \leq \eta$ provided $E \ni \varepsilon \leq \alpha$.

We express this by writing $u_{\varepsilon} \rightarrow u_{0}$ reiteratively in $L^{p}(\Omega)$-weak $\Sigma$ in case (i), and $u_{\varepsilon} \rightarrow u_{0}$ reiteratively in $L^{p}(\Omega)$-strong $\Sigma$ in case (ii).

There is no difficulty in verifying the following results.

(1) Suppose $u_{0}=\widehat{v}_{0}$ with $v_{0} \in L^{p}(\Omega ; A)$. Then $u_{\varepsilon} \rightarrow u_{0}$ reiteratively in $L^{p}(\Omega)$-strong $\Sigma$ if and only if $\left\|u_{\varepsilon}-v_{0}^{\varepsilon}\right\|_{L^{p}(\Omega)} \rightarrow 0$ as $E \ni \varepsilon \rightarrow 0$.

(2) For $u \in L^{p}(\Omega ; A)$ we have $u^{\varepsilon} \rightarrow \widehat{u}$ reiteratively in $L^{p}(\Omega)$-strong $\Sigma$.

(3) If $u_{\varepsilon} \rightarrow u$ in $L^{p}(\Omega)$ (strong) as $E \ni \varepsilon \rightarrow 0$, then $u_{\varepsilon} \rightarrow u$ reiteratively in $L^{p}(\Omega)$-strong $\Sigma$.

Also, the proof of the next proposition is a simple exercise left to the reader.

Proposition 2.4. Suppose a sequence $\left(u_{\varepsilon}\right)_{\varepsilon \in E} \subset L^{p}(\Omega) \quad(1 \leq p<\infty)$ weakly $\Sigma$-converges reiteratively in $L^{p}(\Omega)$ to some $u_{0} \in L^{p}(\Omega \times \Delta(A))$. Define $u_{0}^{*} \in L^{p}\left(\Omega \times \Delta\left(A_{y}\right)\right)$ as $u_{0}^{*}(x, s)=\int_{\Delta\left(A_{z}\right)} u_{0}(x, s, r) d \beta_{z}(r) \quad(x \in \Omega$, 
$\left.s \in \Delta\left(A_{y}\right)\right)$, and $\widetilde{u} \in L^{p}(\Omega)$ as $\widetilde{u}(x)=\int_{\Delta\left(A_{z}\right)} \int_{\Delta\left(A_{y}\right)} u_{0}(x, s, r) d \beta_{y}(s) d \beta_{z}(r)$ $(x \in \Omega)$. Then, as $E \ni \varepsilon \rightarrow 0$,

(i) $u_{\varepsilon} \rightarrow u_{0}^{*}$ in $L^{p}(\Omega)-$ weak $\Sigma_{y}[18$, Definition 4.1$]$

(ii) $u_{\varepsilon} \rightarrow \widetilde{u}_{0}$ in $L^{p}(\Omega)$-weak.

The results of the $\Sigma$-convergence setting [18] carry over mutatis mutandis, together with their proofs, to the present setting. Let us state the most important of such results.

Proposition 2.5. Assume that $1<p<\infty$. Given a fundamental sequence $E$ and a sequence $\left(u_{\varepsilon}\right)_{\varepsilon \in E}$ which is bounded in $L^{p}(\Omega), a$ subsequence $E^{\prime}$ can be extracted from $E$ such that the sequence $\left(u_{\varepsilon}\right)_{\varepsilon \in E^{\prime}}$ weakly $\Sigma$-converges reiteratively in $L^{p}(\Omega)$.

Proposition 2.6. Suppose a sequence $\left(u_{\varepsilon}\right)_{\varepsilon \in E}$ strongly $\Sigma$-converges reiteratively in $L^{p}(\Omega)$ to some $u_{0} \in L^{p}(\Omega \times \Delta(A))$. Then, as $E \ni \varepsilon \rightarrow 0$,

(i) $u_{\varepsilon} \rightarrow u_{0}$ reiteratively in $L^{p}(\Omega)-$ weak $\Sigma$

(ii) $\left\|u_{\varepsilon}\right\|_{L^{p}(\Omega)} \rightarrow\left\|u_{0}\right\|_{L^{p}(\Omega \times \Delta(A))}$.

Reciprocally, if $p=2$ and if assertions (i)-(ii) hold, then $u_{\varepsilon} \rightarrow u_{0}$ reiteratively in $L^{p}(\Omega)$-strong $\Sigma$.

Proposition 2.7. Suppose the two real numbers $p, q \geq 1$ are such that $\frac{1}{\sigma}=\frac{1}{p}+\frac{1}{q} \leq 1$. Let $u_{0} \in L^{p}(\Omega \times \Delta(A))$ and $v_{0} \in L^{q}(\Omega \times \Delta(A))$, and let $u_{\varepsilon} \in L^{p}(\Omega)$ and $v_{\varepsilon} \in L^{q}(\Omega)$ for $\varepsilon \in E$. Finally, assume that $u_{\varepsilon} \rightarrow u_{0}$ reiteratively in $L^{p}(\Omega)$-strong $\Sigma$ and $v_{\varepsilon} \rightarrow v_{0}$ reiteratively in $L^{q}(\Omega)$-weak $\Sigma$. Then $u_{\varepsilon} v_{\varepsilon} \rightarrow u_{0} v_{0}$ reiteratively in $L^{\sigma}(\Omega)$-weak $\Sigma$.

The notion of a $W^{1, p}(\Omega)$-proper reiteration $H$-structure will play a fundamental role in this study. We refer to, e.g., [1] for the classical Sobolev space $W^{1, p}(\Omega)$, to [19] (see also [18]) for special Sobolev spaces such as $W^{1, p}\left(\Delta\left(A_{y}\right)\right)$ and $W_{\#}^{1, p}\left(\Delta\left(A_{y}\right)\right)$ together with the various associated derivative operators.

Definition 2.3. The reiteration $H$-structure $\Sigma=\Sigma_{y} \times \Sigma_{z}$ is termed $W^{1, p}(\Omega)$-proper ( $p$ a given real number with $p \geq 1$ ) if the following three conditions are satisfied.

$(\mathrm{PR})_{1} \quad \Sigma_{y}$ is total for $p$, i.e., $\mathcal{D}\left(\Delta\left(A_{y}\right)\right)$ is dense in $W^{1, p}\left(\Delta\left(A_{y}\right)\right)$

$(\mathrm{PR})_{2} \quad \Sigma_{z}$ is total for $p$

$(\mathrm{PR})_{3}$ Given a fundamental sequence $E$ and a sequence $\left(u_{\varepsilon}\right)_{\varepsilon \in E}$ which is bounded in $W^{1, p}(\Omega)$, there are a subsequence $E^{\prime}$ extracted from 
$E$ and three functions $u_{0} \in W^{1, p}(\Omega), u_{1} \in L^{p}\left(\Omega ; W_{\#}^{1, p} \Delta\left(A_{y}\right)\right)$ and $u_{2} \in L^{p}\left(\Omega ; L^{p}\left(\Delta\left(A_{y}\right) ; W_{\#}^{1, p} \Delta\left(A_{z}\right)\right)\right)$, such that, as $E^{\prime} \ni \varepsilon \rightarrow 0$,

$$
u_{\varepsilon} \rightarrow u_{0} \text { in } W^{1, p}(\Omega) \text {-weak }
$$

$\frac{\partial u_{\varepsilon}}{\partial x_{j}} \rightarrow \frac{\partial u_{0}}{\partial x_{j}}+\partial_{j} u_{1}+\partial_{j} u_{2}$ reit.in $L^{p}(\Omega)-$ weak $\Sigma(1 \leq j \leq N)$,

where reit. stands for reiteratively.

Our next purpose is to present a few examples of $W^{1, p}(\Omega)$-proper reiteration $H$-structures.

Example 2.7. Our goal here is to show that the reiteration $H$-structure $\Sigma=\Sigma_{\mathbb{Z}^{N}} \times \Sigma_{\mathbb{Z}^{N}}$ of Example 2.1 is $W^{1, p}(\Omega)$-proper for any arbitrary real $p>1$. Let us first recall that when dealing with periodic $H$ structures, it is possible to do without the Gelfand representation theory (see, e.g., [19, Example 3.1]). So, let $Y=(0,1)^{N}$ (the open unit cube in $\mathbb{R}_{y}^{N}$ ) and $Z=(0,1)^{N}$ (the open unit cube in $\mathbb{R}_{z}^{N}$ ). We denote by $\mathcal{C}_{\text {per }}(Y \times Z)$ the space of functions $\psi \in \mathcal{C}\left(\mathbb{R}_{y}^{N} \times \mathbb{R}_{z}^{N}\right)$ that are $Y \times Z$ periodic, i.e., that verify $\psi(y+k, z+\ell)=\psi(y, z)$ for $(y, z) \in \mathbb{R}^{N} \times \mathbb{R}^{N}$ and $(k, \ell) \in \mathbb{Z}^{N} \times \mathbb{Z}^{N}$. Note that $\mathcal{C}_{\text {per }}(Y \times Z)=\mathcal{J}(\Sigma)$ (the image of $\left.\Sigma=\Sigma_{\mathbb{Z}^{N}} \times \Sigma_{\mathbb{Z}^{N}}\right)$. On the other hand, we need the space $L_{p e r}^{p}(Y \times Z)$ of $Y \times Z$-periodic functions in $L_{\text {loc }}^{p}\left(\mathbb{R}_{y}^{N} \times \mathbb{R}_{z}^{N}\right)$, and the space $\mathcal{V}_{\text {div }}(Y \times Z)$ of those $\mathbf{u} \in \mathcal{C}_{\text {per }}^{\infty}(Y \times Z)^{N}$ such that $\operatorname{div}_{y} \mathbf{u}=0$ and $\operatorname{div}_{z} \mathbf{u}=0$, where $\mathcal{C}_{\text {per }}^{\infty}(Y \times Z)=\mathcal{C}_{\text {per }}(Y \times Z) \cap \mathcal{C}^{\infty}\left(\mathbb{R}_{y}^{N} \times \mathbb{R}_{z}^{N}\right)$. As a preliminary step, we have the following

Lemma 2.2. Assume that $1<p<\infty$. Let $\mathbf{f}=\left(f_{j}\right) \in L_{p e r}^{p}(Y \times Z)^{N}$. Suppose we have

$$
\sum_{j=1}^{N} \iint_{Y \times Z} f_{j} v_{j} d y d z=0
$$

for all $\mathbf{v}=\left(v_{j}\right) \in \mathcal{V}_{\text {div }}(Y \times Z)$. Then, there exists a unique couple of functions $u_{1} \in W_{\#}^{1, p}(Y)=\left\{v \in W_{\text {loc }}^{1, p}\left(\mathbb{R}_{y}^{N}\right): v Y\right.$-periodic, $\left.\int_{Y} v(y) d y=0\right\}$, $u_{2} \in L^{p}\left(Y ; W_{\#}^{1, p}(Z)\right)$, such that $\mathbf{f}=D_{y} u_{1}+D_{z} u_{2}$.

Proof. This is a simple adaptation of the proof of [19, Lemma 3.4].

We are now able to state the desired result.

Proposition 2.8. The reiteration $H$-structure $\Sigma=\Sigma_{\mathbb{Z}^{N}} \times \Sigma_{\mathbb{Z}^{N}}$ is $W^{1, p}(\Omega)$-proper for each real $p>1$. 
Proof. Properties (PR $)_{1}-(\mathrm{PR})_{2}$ of Definition 2.3 follow by [19, Proposition 3.2], and (PR $)_{3}$ follows by a classical approach using Lemma 2.2 (see, e.g., [16, Theorem 13]).

Example 2.8. The aim here is to verify that the reiteration $H$-structure $\Sigma=\Sigma_{\mathcal{R}_{y}} \times \Sigma_{\mathcal{R}_{z}}$ of Example 2.2 is $W^{1,2}(\Omega)$-proper. The first step will be to frame a preliminary lemma analogous to Lemma 2.2. For $\mathbf{u}=\left(u_{i}\right) \in \mathcal{D}(\Delta(A))^{N}=\mathcal{G}\left(A^{\infty}\right)^{N}$, where $\mathcal{G}$ is the Gelfand transformation on $A$ (we recall that $\mathcal{G}$ maps $A^{\infty}$ isomorphically over $\mathcal{D}(\Delta(A))$ ), let $\widehat{\operatorname{div}}_{y} \mathbf{u}=\mathcal{G}\left(\operatorname{div}_{y} \mathcal{G}^{-1}(\mathbf{u})\right)$, and $\widehat{\operatorname{div}}_{z} \mathbf{u}=\mathcal{G}\left(\operatorname{div}_{z} \mathcal{G}^{-1}(\mathbf{u})\right)$, where $\mathcal{G}^{-1}(\mathbf{u})=$ $\left(\mathcal{G}^{-1} u_{i}\right)_{1 \leq i \leq N}$.

Lemma 2.3. Let $\mathbf{f}=\left(f_{j}\right) \in L^{2}(\Delta(A))^{N}$. Suppose

$$
\sum_{j=1}^{N} \int_{\Delta(A)} f_{j} v_{j} d \beta=0
$$

for all $\mathbf{v}=\left(v_{j}\right) \in \mathcal{D}(\Delta(A))^{N}$ with $\widehat{\operatorname{div}}_{y} \mathbf{v}=0$ and $\widehat{\operatorname{div}}_{z} \mathbf{v}=0$. There exists a couple of functions $u_{1} \in W_{\#}^{1,2}\left(\Delta\left(A_{y}\right)\right), u_{2} \in L^{2}\left(\Delta\left(A_{y}\right) ; W_{\#}^{1,2}\left(\Delta\left(A_{z}\right)\right)\right)$ such that $\mathbf{f}=\partial_{s} u_{1}+\partial_{r} u_{2}$, where $\partial_{s} u_{1}=\left(\partial_{i} u_{1}\right)_{1 \leq i \leq N}$ and $\partial_{r} u_{2}=\left(\partial_{i} u_{2}\right)_{1 \leq i \leq N}$.

Proof. This follows in view of the proof of [17, Lemma 4.2].

This leads to the next proposition.

Proposition 2.9. The reiteration $H$-structure $\Sigma=\Sigma_{\mathcal{R}_{y}} \times \Sigma_{\mathcal{R}_{z}}$ is $W^{1,2}(\Omega)$-proper.

Proof. Properties $(\mathrm{PR})_{1}-(\mathrm{PR})_{2}$ of Definition 2.3 follow by [17, Proposition 4.3] whereas $(\mathrm{PR})_{3}$ is deduced from Lemma 2.3 in a classical way (see, e.g., the proof of [17, Theorem 4.1]).

Further useful examples of $W^{1, p}(\Omega)$-proper reiteration $H$-structures can be deduced from Examples 2.7 and 2.8 by means of the following lemma.

Lemma 2.4. Consider a further reiteration $H$-structure $\Sigma_{2}=\Sigma_{2 y} \times \Sigma_{2 z}$ on $\mathbb{R}_{y}^{N} \times \mathbb{R}_{z}^{N}$ for $\mathcal{H}^{*}$, where $\Sigma_{2 y}$ (resp. $\Sigma_{2 z}$ ) is a further $H$-structure of class $\mathcal{C}^{\infty}$ on $\mathbb{R}_{y}^{N}$ (resp. $\mathbb{R}_{z}^{N}$ ) for $\mathcal{H}$ (resp. $\mathcal{H}^{\prime}$ ). Suppose each of the couples $\left(\Sigma_{y}, \Sigma_{2 y}\right)$ and $\left(\Sigma_{z}, \Sigma_{2 z}\right)$ satisfies a hypothesis similar to hypothesis $(H)$ of $\left[19\right.$, Section 3]. If the reiteration $H$-structure $\Sigma_{2}$ is $W^{1, p}(\Omega)$-proper for some real $p \geq 1$, then so also is $\Sigma$. 
Proof. Assuming that $\Sigma_{2}$ is $W^{1, p}(\Omega)$-proper for some given real $p \geq 1$, we see immediately by [19, Lemma 3.1] that conditions $(\mathrm{PR})_{1}-(\mathrm{PR})_{2}$ of Definition 2.3 are fulfilled. Thus, it remains to verify $(\mathrm{PR})_{3}$. But this follows by an adaptation of a well-known line of argument (see, e.g., [19, Theorem 3.1]).

We are now able to provide further examples of $W^{1, p}(\Omega)$-proper reiteration $H$-structures.

Example 2.9. The $H$-structure of Example 2.5 is $W^{1, p}(\Omega)$-proper for $p=2$ in general, for any real $p>1$ in the particular case where $\mathcal{R}_{y}=\mathbb{Z}^{N}$ and $\mathcal{R}_{z}=\mathbb{Z}^{N}$. Indeed, this follows by Lemma 2.4 with $\Sigma=\Sigma_{\mathcal{R}_{y}} \times \Sigma_{\infty, \mathcal{R}_{z}}$, $\Sigma_{2}=\Sigma_{\mathcal{R}_{y}} \times \Sigma_{\mathcal{R}_{z}}$, and use of Propositions 2.8-2.9 and the fact that the couple $\left\{\Sigma_{\mathcal{R}_{z}}, \Sigma_{\infty, \mathcal{R}_{z}}\right\}$ satisfies hypothesis $(H)$ (see $\left.[18,19]\right)$.

Example 2.10. Let $\mathcal{R}_{y}$ be a countable subgroup of $\mathbb{R}_{y}^{N}$. The reiteration $H$-structure $\Sigma=\Sigma_{\mathcal{R}_{y}} \times \Sigma_{\infty}$ (Example 2.4) is $W^{1,2}(\Omega)$-proper. This follows by the same argument as used to prove [18, Corollary 4.4 and Example 4.4]. The details are left to the reader.

Example 2.11. The reiteration $H$-structure of Example 2.6 is $W^{1, p}(\Omega)$ proper for $p=2$ in general, and for any $p>1$ in the particular case when $\mathcal{R}_{y}=\mathbb{Z}^{N}, \mathcal{R}_{z}=\mathbb{Z}^{N}$. Indeed, this follows by applying Lemma 2.4 with $\Sigma=\Sigma_{\infty, \mathcal{R}_{y}} \times \Sigma_{\infty, \mathcal{R}_{z}}, \Sigma_{2}=\Sigma_{\mathcal{R}_{y}} \times \Sigma_{\mathcal{R}_{z}}$, and proceeding as in Example 2.9.

Example 2.12. The $H$-structure of Example 2.3 is $W^{1,2}(\Omega)$-proper. Indeed, since $\Sigma_{\infty}=\Sigma_{\infty,\{\omega\}}$, this is nothing else but a particular case of Example 2.11.

\section{The abstract homogenization problem}

3.1. Setting of the abstract problem and preliminaries. Before we can state the abstract homogenization problem for (1.1) we need further details. The basic notation is as in Section 2. Let $1 \leq p<\infty$. We denote by $\Xi^{p}\left(\mathbb{R}_{y}^{N} ; \mathcal{B}\left(\mathbb{R}_{z}^{N}\right)\right)$, or simply $\Xi^{p}$ when there is no danger of confusion, the space of those functions $u \in L_{l o c}^{p}\left(\mathbb{R}_{y}^{N} ; \mathcal{B}\left(\mathbb{R}_{z}^{N}\right)\right)$ for which

$$
\sup _{0<\varepsilon \leq 1} \int_{U}\left\|u\left(\frac{x}{\varepsilon}, \cdot\right)\right\|_{\infty}^{p} d x<\infty
$$

for any bounded open set $U$ in $\mathbb{R}_{x}^{N}$. This is a Banach space with norm

$$
\|u\|_{\Xi^{p}}=\sup _{0<\varepsilon \leq 1}\left(\int_{B_{N}}\left\|u\left(\frac{x}{\varepsilon}, \cdot\right)\right\|_{\infty}^{p} d x\right)^{\frac{1}{p}} \quad\left(u \in \Xi^{p}\right)
$$


where $B_{N}$ is the open unit ball in $\mathbb{R}_{x}^{N}$.

Remark 3.1. It should be noted that the way we defined $\psi^{\varepsilon}$ (in (2.2)) for $\psi \in L^{\infty}\left(\mathbb{R}_{y}^{N} ; \mathcal{B}\left(\mathbb{R}_{z}^{N}\right)\right)$ remains rigorously valid when $L^{\infty}\left(\mathbb{R}_{y}^{N} ; \mathcal{B}\left(\mathbb{R}_{z}^{N}\right)\right)$ is replaced by $L_{\text {loc }}^{p}\left(\mathbb{R}_{y}^{N} ; \mathcal{B}\left(\mathbb{R}_{z}^{N}\right)\right)$, so that $(2.2)$ extends to all $\psi \in$ $L_{\text {loc }}^{p}\left(\mathbb{R}_{y}^{N} ; \mathcal{B}\left(\mathbb{R}_{z}^{N}\right)\right)$. Furthermore, for each bounded open set $U \subset \mathbb{R}_{x}^{N}$, we have

$$
\sup _{0<\varepsilon \leq 1}\left(\int_{U}\left|u\left(\frac{x}{\varepsilon}, \frac{x}{\varepsilon^{2}}\right)\right|^{p} d x\right)^{\frac{1}{p}} \leq n^{\frac{N}{p}}\|u\|_{\Xi^{p}} \quad\left(u \in \Xi^{p}\right),
$$

where $n$ is some positive integer such that $U \subset n B_{N}$. Thus, as $\varepsilon$ ranges over $(0,1)$, the mappings $u \rightarrow u^{\varepsilon}$ are uniformly equicontinuous from $\Xi^{p}$ into $L_{l o c}^{p}\left(\mathbb{R}_{x}^{N}\right)$.

Now, in the notation of Subsection 2.2, let $\Sigma=\Sigma_{y} \times \Sigma_{z}$ be a reiteration $H$-structure on $\mathbb{R}_{y}^{N} \times \mathbb{R}_{z}^{N}$ for $\mathcal{H}^{*}$. We define $\mathfrak{X}_{\Sigma}^{p}\left(\mathbb{R}_{y}^{N} ; \mathcal{B}\left(\mathbb{R}_{z}^{N}\right)\right.$ ) (or $\mathfrak{X}_{\Sigma}^{p}$, or simply $\mathfrak{X}^{p}$ when there is no danger of confusion) to be the closure of $A=\mathcal{J}(\Sigma)$ in $\Xi^{p}$. We provide $\mathfrak{X}_{\Sigma}^{p}$ with the $\Xi^{p}$-norm, which makes it a Banach space. Propositions 2.3-2.4 and Corollaries 2.1-2.2 of [18] carry over mutatis mutandis, together with their proofs, to the present context. Let us especially attract attention to the following two fundamental propositions.

Proposition 3.1. The mean value $M$ on $\mathbb{R}^{N} \times \mathbb{R}^{N}$ for $\mathcal{H}^{*}$, viewed as defined on $A=\mathcal{J}(\Sigma)$, extends by continuity to a positive continuous linear form (still denoted by $M$ ) on $\mathfrak{X}_{\Sigma}^{p}$. Furthermore, for each $u \in \mathfrak{X}_{\Sigma}^{p}$, we have $u^{\varepsilon} \rightarrow M(u)$ in $L^{p}(\Omega)$-weak as $\varepsilon \rightarrow 0$, where $u^{\varepsilon}$ (defined as in (2.2)) is considered as a function in $L^{p}(\Omega)$, and $\Omega$ is any fixed bounded open set in $\mathbb{R}_{x}^{N}$.

Proposition 3.2. The Gelfand transformation $\mathcal{G}: A \rightarrow \mathcal{C}(\Delta(A))$ extends by continuity to a (unique) continuous linear mapping, still denoted by $\mathcal{G}$, of $\mathfrak{X}_{\Sigma}^{p}$ into $L^{p}(\Delta(A))$.

Remark 3.2. As a direct consequence of the preceding propositions, we have $M(u)=\int_{\Delta(A)} \mathcal{G}(u) d \beta$ for all $u \in \mathfrak{X}_{\Sigma}^{p}$.

At the present time, let $\mathfrak{X}_{\Sigma}^{p, \infty}=\mathfrak{X}_{\Sigma}^{p} \cap L^{\infty}\left(\mathbb{R}_{y}^{N} ; \mathcal{B}\left(\mathbb{R}_{z}^{N}\right)\right)$ be provided with the $L^{\infty}\left(\mathbb{R}_{y}^{N} ; \mathcal{B}\left(\mathbb{R}_{z}^{N}\right)\right)$-norm. It can be shown (as in part (v) of [18, Corollary 2.2]) that for $u \in \mathfrak{X}_{\Sigma}^{p, \infty}$, we have $\mathcal{G}(u) \in L^{\infty}(\Delta(A))$ and $\|\mathcal{G}(u)\|_{L^{\infty}(\Delta(A))} \leq\|u\|_{L^{\infty}\left(\mathbb{R}_{y}^{N} ; \mathcal{B}\left(\mathbb{R}_{z}^{N}\right)\right)}$. Another result worth mentioning is the following, where $\Omega$ is as in Proposition 3.1. 
Proposition 3.3. Let $1<p<\infty$. Let $E$ be a fundamental sequence. Suppose a sequence $\left(u_{\varepsilon}\right)_{\varepsilon \in E}$ weakly $\Sigma$-converges reiteratively in $L^{p}(\Omega)$ to some $u_{0} \in L^{p}(\Omega \times \Delta(A)$ ) (Definition 2.2). Then, as $E \ni \varepsilon \rightarrow 0$, (2.6) holds for $v \in \mathcal{C}\left(\bar{\Omega} ; \mathfrak{X}_{\Sigma}^{p^{\prime}, \infty}\right)\left(p^{\prime}=\frac{p}{p-1}\right)$.

Proof. There is no difficulty in showing that (2.6) holds for $v \in$ $\mathcal{C}(\bar{\Omega}) \otimes \mathfrak{X}_{\Sigma}^{p^{\prime}, \infty}$. Hence the desired result follows by mere routine and use of the density of $\mathcal{C}(\bar{\Omega}) \otimes \mathfrak{X}_{\Sigma}^{p^{\prime}, \infty}$ in $\mathcal{C}\left(\bar{\Omega} ; \mathfrak{X}_{\Sigma}^{p^{\prime}, \infty}\right)$.

The next result is a direct consequence of Proposition 3.3.

Corollary 3.1. Let the hypotheses be as in Proposition 3.3. If $v \in$ $\mathcal{C}\left(\bar{\Omega} ; \mathfrak{X}_{\Sigma}^{p^{\prime}, \infty}\right)$, then $v^{\varepsilon} \rightarrow \widehat{v}$ reiteratively in $L^{p}(\Omega)$-weak $\Sigma$ as $E \ni \varepsilon \rightarrow 0$.

We turn now to the statement of the abstract homogenization problem for (1.1).

Throughout the remainder of the present section it is assumed that $1<p<\infty$. Our main goal is to investigate the limiting behaviour, as $\varepsilon \rightarrow 0$, of $u_{\varepsilon}$ (the solution of (1.1) for fixed $\varepsilon>0$ ) under the abstract hypothesis

$$
a_{i}(\cdot, \cdot, \psi) \in \mathfrak{X}_{\Sigma}^{p^{\prime}}\left(\mathbb{R}_{y}^{N} ; \mathcal{B}\left(\mathbb{R}_{z}^{N}\right)\right) \text { for all } \psi \in\left(A_{\mathbb{R}}\right)^{N} \quad(1 \leq i \leq N)
$$

where $p^{\prime}=\frac{p}{p-1}$ and

$$
A_{\mathbb{R}}=A \cap \mathcal{C}\left(\mathbb{R}_{y}^{N} \times \mathbb{R}_{z}^{N} ; \mathbb{R}\right) \text { with } A=\mathcal{J}(\Sigma),
$$

where $a_{i}(\cdot, \cdot, \psi)$ stands for the function $(y, z) \rightarrow a_{i}(y, z, \psi(y, z))$ from $\mathbb{R}^{N} \times \mathbb{R}^{N}$ into $\mathbb{R}$.

This is referred to as the homogenization problem for (1.1) in the abstract deterministic setting associated with the reiteration $H$-structure $\Sigma=\Sigma_{y} \times \Sigma_{z}$.

The resolution of this problem requires a few preliminaries. We start with one fundamental lemma.

Lemma 3.1. Let (3.1) hold. Then, $a_{i}(\cdot, \cdot, \psi) \in L^{\infty}\left(\mathbb{R}_{y}^{N} ; A_{z}\right) \quad(1 \leq i \leq$ $N)$ for any $\psi \in\left(A_{\mathbb{R}}\right)^{N}$.

Proof. Thanks to part (iii) of (1.4), the lemma is proved if we can check that for $\psi \in\left(A_{\mathbb{R}}\right)^{N}$ and $1 \leq i \leq N$, the function $a_{i}(\cdot, \cdot, \psi)$ is measurable from $\mathbb{R}_{y}^{N}$ into $A_{z}$. Thus, in view of (3.1), it is enough to establish that

$$
\mathfrak{X}_{\Sigma}^{q}\left(\mathbb{R}_{y}^{N} ; \mathcal{B}\left(\mathbb{R}_{z}^{N}\right)\right) \subset L_{l o c}^{q}\left(\mathbb{R}_{y}^{N} ; A_{z}\right)(1 \leq q<\infty) .
$$


But for any fixed real $q \geq 1$, we clearly have $A_{y} \otimes A_{z} \subset L_{l o c}^{q}\left(\mathbb{R}_{y}^{N} ; A_{z}\right)$, where $A_{y}=\mathcal{J}\left(\Sigma_{y}\right)$ and $A_{z}=\mathcal{J}\left(\Sigma_{z}\right)$, of course. Hence (3.2) follows by three facts : 1) $A_{y} \otimes A_{z}$ is dense in $\mathfrak{X}_{\Sigma}^{q}\left(\mathbb{R}_{y}^{N} ; \mathcal{B}\left(\mathbb{R}_{z}^{N}\right)\right)$, as a consequence of the fact that $A_{y} \otimes A_{z}$ is dense in $A$ [18, Proposition 3.2];2) $\Xi^{q}\left(\mathbb{R}_{y}^{N} ; \mathcal{B}\left(\mathbb{R}_{z}^{N}\right)\right)$ is continuously embedded in $\left.L_{\text {loc }}^{q}\left(\mathbb{R}_{y}^{N} ; \mathcal{B}\left(\mathbb{R}_{z}^{N}\right)\right) ; 3\right) L_{\text {loc }}^{q}\left(\mathbb{R}_{y}^{N} ; A_{z}\right)$ is closed in $L_{l o c}^{q}\left(\mathbb{R}_{y}^{N} ; \mathcal{B}\left(\mathbb{R}_{z}^{N}\right)\right)$.

Remark 3.3. Lemma 3.1 shows immediately that (3.1) implies (2.5).

At the present time, let the index $1 \leq i \leq N$ be fixed. For $\varphi=\left(\varphi_{j}\right)_{1 \leq j \leq N}$ in $\mathcal{C}_{\mathbb{R}}(\Delta(A))^{N}$, where $\mathcal{C}_{\mathbb{R}}(\Delta(A))=\mathcal{C}(\Delta(A) ; \mathbb{R})$, let

$$
b_{i}(\varphi)=\mathcal{G}\left(a_{i}\left(\cdot, \cdot, \mathcal{G}^{-1} \varphi\right)\right)
$$

where $\mathcal{G}^{-1} \varphi=\left(\mathcal{G}^{-1} \varphi_{j}\right)_{1 \leq j \leq N}$. Assuming (3.1), we see by Lemma 3.1 that $a_{i}\left(\cdot, \cdot, \mathcal{G}^{-1} \varphi\right)$ lies in $\mathfrak{X}_{\Sigma}^{p^{\prime}, \infty}\left(\mathbb{R}_{y}^{N} ; \mathcal{B}\left(\mathbb{R}_{z}^{N}\right)\right)$; hence (3.3) defines a mapping $b_{i}$ of $\mathcal{C}_{\mathbb{R}}(\Delta(A))^{N}$ into $L^{\infty}(\Delta(A))$. The next proposition and corollary can be achieved using Proposition 2.2 and Corollary 3.1 and reasoning as in the proof of [19, Proposition 4.1 and Corollary 4.1].

Proposition 3.4. Let $1<p<\infty$. Let $\Omega$ be a bounded open set in $\mathbb{R}_{x}^{N}$. Suppose (3.1) holds. Then the following assertions are true:

(i) Let the index $1 \leq i \leq N$ be fixed. For $\psi=\left(\psi_{j}\right)_{1 \leq j \leq N}$ in $\mathcal{C}\left(\bar{\Omega} ;\left(A_{\mathbb{R}}\right)^{N}\right)$, the function $b_{i} \circ \widehat{\psi}$ (usual composition) of $\bar{\Omega}$ into $L^{\infty}(\Delta(A))$ lies in $\mathcal{C}\left(\bar{\Omega} ; L^{\infty}(\Delta(A))\right)$ and further $a_{i}^{\varepsilon}\left(\cdot, \cdot, \psi^{\varepsilon}\right) \rightarrow b_{i} \circ \widehat{\psi}$ reit. in $L^{p^{\prime}}(\Omega)$ weak $\Sigma$ as $E \ni \varepsilon \rightarrow 0$, where $\psi^{\varepsilon}=\left(\psi_{j}^{\varepsilon}\right)_{1 \leq j \leq N}$ ( $\psi_{j}^{\varepsilon}$ defined as in $\left.(2.1)\right)$.

(ii) The mapping $\phi \rightarrow b(\phi)=\left(b_{i} \circ \phi\right)_{1 \leq i \leq N}$ of $\mathcal{C}\left(\bar{\Omega} ; \mathcal{C}_{\mathbb{R}}(\Delta(A))^{N}\right)$ into $L^{p^{\prime}}(\Omega \times \Delta(A))^{N}$ extends by continuity to a mapping, still denoted by $b$, of $L^{p}\left(\Omega ; L_{\mathbb{R}}^{p}(\Delta(A))^{N}\right)$ into $L^{p^{\prime}}(\Omega \times \Delta(A))^{N}$ such that

$$
\begin{aligned}
\| b(\mathbf{u}) & -b(\mathbf{v}) \|_{L^{p^{\prime}}(\Omega \times \Delta(A))^{N}} \\
& \leq c_{1}\|1+|\mathbf{u}|+|\mathbf{v}|\|_{L^{p}(\Omega \times \Delta(A))}^{p-1-\alpha_{1}}\|\mathbf{u}-\mathbf{v}\|_{L^{p}\left(\Omega ; L^{p}(\Delta(A))^{N}\right)}^{\alpha_{1}}
\end{aligned}
$$

and

$$
\begin{aligned}
& (b(\mathbf{u})-b(\mathbf{v})) \cdot(\mathbf{u}-\mathbf{v}) \\
& \quad \geq c_{2}(1+|\mathbf{u}|+|\mathbf{v}|)^{p-\alpha_{2}}|\mathbf{u}-\mathbf{v}|^{\alpha_{2}} \text { a.e. in } \Omega \times \Delta(A)
\end{aligned}
$$

for all $\mathbf{u}, \mathbf{v} \in L^{p}\left(\Omega ; L_{\mathbb{R}}^{p}(\Delta(A))^{N}\right)$.

Corollary 3.2. Let the hypotheses be those of Proposition 3.4. For each real $\varepsilon>0$, Let $\phi_{\varepsilon} \in \mathcal{D}_{\mathbb{R}}(\Omega)=\mathcal{C}_{0}^{\infty}(\Omega ; \mathbb{R})$ be given by 


$$
\phi_{\varepsilon}(x)=\psi_{0}(x)+\varepsilon \psi_{1}\left(x, \frac{x}{\varepsilon}\right)+\varepsilon^{2} \psi_{2}\left(x, \frac{x}{\varepsilon}, \frac{x}{\varepsilon^{2}}\right) \quad(x \in \Omega)
$$

with $\psi_{0} \in \mathcal{D}_{\mathbb{R}}(\Omega), \psi_{1} \in \mathcal{D}_{\mathbb{R}}(\Omega) \otimes \mathbb{R}_{y} A_{y}^{\infty}, \psi_{2} \in \mathcal{D}_{\mathbb{R}}(\Omega) \otimes \mathbb{R}_{y} A_{y}^{\infty} A_{\mathbb{R}}^{\infty}$, where $\mathbb{R}_{y}^{\infty}=A_{y}^{\infty} \cap \mathcal{C}\left(\mathbb{R}^{N} ; \mathbb{R}\right)$ and a similar definition for $\mathbb{R}_{z}^{\infty}$. Let the index $1 \leq i \leq N$ be fixed. Then, as $\varepsilon \rightarrow 0$,

$$
a_{i}^{\varepsilon}\left(\cdot, \cdot, D \phi_{\varepsilon}\right) \rightarrow b_{i}\left(D \psi_{0}+\partial_{s} \widehat{\psi}_{1}+\partial_{r} \widehat{\psi}_{2}\right) \text { reit. in } L^{p^{\prime}}(\Omega)-\text { weak } \Sigma,
$$

where : $\partial_{s} \widehat{\psi}_{1}=\left(\partial_{j} \widehat{\psi}_{1}\right)_{1 \leq j \leq N}$ with $\partial_{j} \widehat{\psi}_{1}=\partial_{j} \circ \widehat{\psi}_{1}$, the partial derivative $\partial_{j}$ being here taken on $\Delta(A)=\Delta\left(A_{y}\right) \times \Delta\left(A_{z}\right)$ with respect to $\Delta\left(A_{y}\right)$; $\partial_{r} \widehat{\psi}_{2}=\left(\partial_{j} \widehat{\psi}_{2}\right)_{1 \leq j \leq N}$ with $\partial_{j} \widehat{\psi}_{2}=\partial_{j} \circ \widehat{\psi}_{2}, \partial_{j}$ being this time taken on $\Delta(A)$ with respect to $\Delta\left(A_{z}\right)$; the functions $\widehat{\psi}_{1}$ and $\widehat{\psi}_{2}$ are viewed as defined on $\bar{\Omega}$ with values in $\mathcal{D}(\Delta(A))$.

Furthermore, if $\left(v_{\varepsilon}\right)_{\varepsilon \in E}$ is a sequence in $L^{p}(\Omega)$ such that $v_{\varepsilon} \rightarrow v_{0}$ reit. in $L^{p}(\Omega)$-weak $\Sigma$ as $E \ni \varepsilon \rightarrow 0$, then, as $E \ni \varepsilon \rightarrow 0$,

$$
\int_{\Omega} a_{i}^{\varepsilon}\left(\cdot, \cdot, D \phi_{\varepsilon}\right) v_{\varepsilon} d x \rightarrow \iint_{\Omega \times \Delta(A)} b_{i}\left(D \psi_{0}+\partial_{s} \widehat{\psi}_{1}+\partial_{r} \widehat{\psi}_{2}\right) v_{0} d x d \beta .
$$

Remark 3.4. Let $\varphi \in \mathcal{C}^{1}(\Delta(A))$. By $\partial_{j} \varphi$ to be the partial derivative (of index $j$ ) on $\Delta(A)=\Delta\left(A_{y}\right) \times \Delta\left(A_{z}\right)$ with respect to $\Delta\left(A_{y}\right)$ we mean the function $(s, r) \rightarrow\left[\partial_{j} \varphi(\cdot, r)\right](s)$ of $\Delta(A)$ into $\mathbb{C}$, where $\partial_{j} \varphi(\cdot, r)$ (for fixed $r \in \Delta\left(A_{z}\right)$ ) denotes the partial derivative (of index $j$ ) of $\varphi(\cdot, r)$ on $\Delta\left(A_{y}\right)$.

3.2. The abstract homogenization result. Let the basic notation be as above. Let $1<p<\infty$. We assume that the reiteration $H$-structure $\Sigma=\Sigma_{y} \times \Sigma_{z}$ is $W^{1, p}(\Omega)$-proper (Definiton 2.3). Let

$$
\begin{aligned}
\mathbb{F}_{0}^{1, p}=W_{0}^{1, p}(\Omega ; \mathbb{R}) \times L^{p}\left(\Omega ; W_{\#}^{1, p}\left(\Delta\left(A_{y}\right) ; \mathbb{R}\right)\right) \\
\times L^{p}\left(\Omega ; L^{p}\left(\Delta\left(A_{y}\right) ; W_{\#}^{1, p}\left(\Delta\left(A_{z}\right) ; \mathbb{R}\right)\right)\right)
\end{aligned}
$$

where

$$
W_{\#}^{1, p}\left(\Delta\left(A_{y}\right) ; \mathbb{R}\right)=\left\{v \in W_{\#}^{1, p}\left(\Delta\left(A_{y}\right)\right): \partial_{j} v \in L_{\mathbb{R}}^{p}\left(\Delta\left(A_{y}\right)\right)(1 \leq j \leq N)\right\}
$$

and an analogous definition for $W_{\#}^{1, p}\left(\Delta\left(A_{z}\right) ; \mathbb{R}\right)$. We provide $\mathbb{F}_{0}^{1, p}$ with the norm

$$
\begin{aligned}
& \|\mathbf{u}\|_{\mathbb{F}_{0}^{1, p}}=\sum_{i=1}^{N}\left[\left\|D_{x_{i}} u_{0}\right\|_{L^{p}(\Omega)}+\left\|\partial_{i} u_{1}\right\|_{L^{p}\left(\Omega \times \Delta\left(A_{y}\right)\right)}+\left\|\partial_{i} u_{2}\right\|_{L^{p}(\Omega \times \Delta(A))}\right] \\
& \mathbf{u}=\left(u_{0}, u_{1}, u_{2}\right) \in \mathbb{F}_{0}^{1, p} \quad\left(\text { with } D_{x_{i}}=\frac{\partial}{\partial x_{i}}\right)
\end{aligned}
$$


which makes it a Banach space. We will need the space

$$
\begin{aligned}
F_{0}^{\infty}=\mathcal{D}_{\mathbb{R}}(\Omega) \times\left[\mathcal{D}_{\mathbb{R}}(\Omega) \otimes\right. & \left.J_{y}\left(\mathcal{D}_{\mathbb{R}}\left(\Delta\left(A_{y}\right)\right) / \mathbb{R}\right)\right] \times \\
& {\left[\mathcal{D}_{\mathbb{R}}(\Omega) \otimes \mathcal{D}_{\mathbb{R}}\left(\Delta\left(A_{y}\right)\right) \otimes J_{z}\left(\mathcal{D}_{\mathbb{R}}\left(\Delta\left(A_{z}\right)\right) / \mathbb{R}\right)\right], }
\end{aligned}
$$

where

$$
\begin{gathered}
\mathcal{D}_{\mathbb{R}}\left(\Delta\left(A_{y}\right)\right)=\mathcal{D}\left(\Delta\left(A_{y}\right)\right) \cap \mathcal{C}\left(\Delta\left(A_{y}\right) ; \mathbb{R}\right), \\
\mathcal{D}_{\mathbb{R}}\left(\Delta\left(A_{y}\right)\right) / \mathbb{C}=\left\{\varphi \in \mathcal{D}_{\mathbb{R}}\left(\Delta\left(A_{y}\right)\right): \int_{\Delta\left(A_{y}\right)} \varphi d \beta_{y}=0\right\},
\end{gathered}
$$

and $J_{y}$ denotes the canonical mapping of $W^{1, p}\left(\Delta\left(A_{y}\right)\right) / \mathbb{C}$ into its separated completion $W_{\#}^{1, p}\left(\Delta\left(A_{y}\right)\right.$ ) (of course, all that remaining valid with $z$ in place of index $y$ ).

In view of $(\mathrm{PR})_{1}-(\mathrm{PR})_{2}$ in Definition 2.3 (use also [19, Remark 3.5]), $F_{0}^{\infty}$ is dense in $\mathbb{F}_{0}^{1, p}$.

Remark 3.5. It amounts to the same thing to define $F_{0}^{\infty}$ as the space of all $\phi=\left(\psi_{0}, J_{y} \circ \widehat{\psi}_{1}, J_{z} \circ \widehat{\psi}_{2}\right)$ with $\psi_{0} \in \mathcal{D}_{\mathbb{R}}(\Omega), \psi_{1} \in \mathcal{D}_{\mathbb{R}}(\Omega) \otimes\left(\mathbb{R} A_{y}^{\infty} / \mathbb{C}\right)$ and $\psi_{2} \in \mathcal{D}_{\mathbb{R}}(\Omega) \otimes \mathbb{R}_{y}^{\infty} \otimes\left(\mathbb{R} A_{z}^{\infty} / \mathbb{C}\right)$, where $:{ }_{\mathbb{R}} A_{y}^{\infty} / \mathbb{C}=\left\{\psi \in \mathbb{R}_{y}^{\infty}: M(\psi)=\right.$ $0\}$ ( $M$ the mean value on $\mathbb{R}_{y}^{N}$ for $\mathcal{H}$ ), ${ }_{\mathbb{R}} A_{z}^{\infty} / \mathbb{C}=\left\{\psi \in \mathbb{R}_{z}^{\infty}: M(\psi)=0\right\}$ ( $M$ the mean value on $\mathbb{R}_{z}^{N}$ for $\mathcal{H}^{\prime}$ ), $\widehat{\psi}_{1}=\mathcal{G} \circ \psi_{1}$ is viewed as a mapping of $\Omega$ into $\mathcal{D}\left(\Delta\left(A_{y}\right)\right)$ and $\widehat{\psi}_{2}=\mathcal{G} \circ \psi_{2}$ as a mapping of $\Omega \times \Delta\left(A_{y}\right)$ into $\mathcal{D}\left(\Delta\left(A_{z}\right)\right)$.

We will also need the variational problem

$$
\begin{aligned}
& \mathbf{u}=\left(u_{0}, u_{1}, u_{2}\right) \in \mathbb{F}_{0}^{1, p}: \\
& \iint_{\Omega \times \Delta(A)} b(\mathbb{D} \mathbf{u}) \cdot \mathbb{D} \mathbf{v} d x d \beta=\left\langle f, v_{0}\right\rangle \\
& \text { for all } \mathbf{v}=\left(v_{0}, v_{1}, v_{2}\right) \in \mathbb{F}_{0}^{1, p}
\end{aligned}
$$

where $f \in W^{-1, p^{\prime}}(\Omega ; \mathbb{R}),\langle$,$\rangle stands for the duality pairing between$ $W^{-1, p^{\prime}}(\Omega ; \mathbb{R})$ and $W_{0}^{1, p}(\Omega ; \mathbb{R})$, and where for each $\mathbf{v}=\left(v_{0}, v_{1}, v_{2}\right)$ in $\mathbb{F}_{0}^{1, p}$, we denote $\mathbb{D} \mathbf{v}=D v_{0}+\partial_{s} v_{1}+\partial_{r} v_{2}$ with $\partial_{s} v_{1}=\left(\partial_{j} v_{1}\right)_{1 \leq j \leq N}$ and $\partial_{r} v_{2}=$ $\left(\partial_{j} v_{2}\right)_{1 \leq j \leq N}$. According to (3.4), there is at most one $\mathbf{u}=\left(u_{0}, u_{1}, u_{2}\right)$ verifying (3.6).

We are now in a position to prove the basic deterministic homogenization theorem for (1.1).

Theorem 3.1. Let $1<p<\infty$. Suppose (3.1) holds and further $\Sigma=\Sigma_{y} \times \Sigma_{z}$ is $W^{1, p}(\Omega)$-proper. For each fixed real number $\varepsilon>0$, let $u_{\varepsilon}$ be the solution of (1.1). Then, as $\varepsilon \rightarrow 0$,

$$
u_{\varepsilon} \rightarrow u_{0} \text { in } W_{0}^{1, p}(\Omega)-w e a k,
$$




$$
\frac{\partial u_{\varepsilon}}{\partial x_{i}} \rightarrow \frac{\partial u_{0}}{\partial x_{i}}+\partial_{i} u_{1}+\partial_{i} u_{2} \quad \text { reit. in } L^{p}(\Omega)-\text { weak } \Sigma(1 \leq i \leq N),
$$

where $\mathbf{u}=\left(u_{0}, u_{1}, u_{2}\right)$ is uniquely defined by (3.6).

Proof. It is an easy matter to check using Corollary 2.1 that the (generalized) sequence $\left(u_{\varepsilon}\right)_{\varepsilon>0}$ is bounded in $W_{0}^{1, p}(\Omega)$. Thus, given an arbitrary fundamental sequence $E$, appeal to the $W^{1, p}(\Omega)$-properness of $\Sigma$ yields a subsequence $E^{\prime}$ from $E$ and some triple $\mathbf{u}=\left(u_{0}, u_{1}, u_{2}\right) \in \mathbb{F}_{0}^{1, p}$ such that (3.7) and (3.8) hold as $E^{\prime} \ni \varepsilon \rightarrow 0$. Therefore, recalling that the variational problem (3.6) admits at most one solution, we see that the theorem is proved if we can show that $\mathbf{u}=\left(u_{0}, u_{1}, u_{2}\right)$ verifies the variational equation in (3.6). For this purpose, let us fix freely $\phi=\left(\psi_{0}, J_{y} \circ \widehat{\psi}_{1}, J_{z} \circ \widehat{\psi}_{2}\right) \in F_{0}^{\infty}$ as in Remark 3.5, and let us attach to $\phi$ the sequence $\left(\phi_{\varepsilon}\right)_{\varepsilon>0}, \phi_{\varepsilon}$ given by (3.5). It is a simple exercise to verify that

$$
\left\langle f, u_{\varepsilon}-\phi_{\varepsilon}\right\rangle-\int_{\Omega} a^{\varepsilon}\left(\cdot, \cdot, D \phi_{\varepsilon}\right) \cdot\left(D u_{\varepsilon}-D \phi_{\varepsilon}\right) d x \geq 0 .
$$

The next step is to pass to the limit in (3.9) when $E^{\prime} \ni \varepsilon \rightarrow 0$. Before we can do this, however, we need to know that as $\varepsilon \rightarrow 0$,

$$
D_{x_{i}} \phi_{\varepsilon} \rightarrow D_{x_{i}} \psi_{0}+\partial_{i} \widehat{\psi}_{1}+\partial_{i} \widehat{\psi}_{2} \text { reit. in } L^{p}(\Omega) \text {-weak } \Sigma(1 \leq i \leq N)
$$

and

$$
\phi_{\varepsilon} \rightarrow \psi_{0} \text { in } W_{0}^{1, p}(\Omega) \text {-weak, }
$$

where $D_{x_{i}}=\frac{\partial}{\partial x_{i}}$. Let us verify this. Considering the obvious equality

$$
D_{x_{i}} \phi_{\varepsilon}=D_{x_{i}} \psi_{0}+\left(D_{y_{i}} \psi_{1}\right)^{\varepsilon}+\left(D_{z_{i}} \psi_{2}\right)^{\varepsilon}+\varepsilon\left[\left(D_{x_{i}} \psi_{1}\right)^{\varepsilon}+\left(D_{y_{i}} \psi_{2}\right)^{\varepsilon}\right]+\varepsilon^{2}\left(D_{x_{i}} \psi_{2}\right)^{\varepsilon}
$$

where the index $i$ is freely fixed and the notation $u^{\varepsilon}$ is as in (2.1), one is immediately led to

$$
\left\|D_{x_{i}} \phi_{\varepsilon}-D_{x_{i}} \psi_{0}-\left(D_{y_{i}} \psi_{1}\right)^{\varepsilon}-\left(D_{z_{i}} \psi_{2}\right)^{\varepsilon}\right\|_{L^{p}(\Omega)} \rightarrow 0
$$

as $\varepsilon \rightarrow 0$. Since $\left(D_{y_{i}} \psi_{1}\right)^{\varepsilon} \rightarrow \partial_{i} \widehat{\psi}_{1}$ reit. in $L^{p}(\Omega)$-strong $\Sigma$ as $\varepsilon \rightarrow 0$ and $\left(D_{z_{i}} \psi_{2}\right)^{\varepsilon} \rightarrow \partial_{i} \widehat{\psi}_{2}$ reit. in $L^{p}(\Omega)$-strong $\Sigma$ as $\varepsilon \rightarrow 0$, we deduce that

$$
D_{x_{i}} \phi_{\varepsilon} \rightarrow D_{x_{i}} \psi_{0}+\partial_{i} \widehat{\psi}_{1}+\partial_{i} \widehat{\psi}_{2} \text { reit. in } L^{p}(\Omega) \text {-strong } \Sigma \text {. }
$$

Hence (3.10) follows by Proposition 2.6, whereas (3.11) follows from (3.10) by [part (ii) of] Proposition 2.4 and use of the fact that $\int_{\Delta(A)}\left(\partial_{i} \widehat{\psi}_{1}+\right.$ 
$\left.\partial_{i} \widehat{\psi}_{2}\right) d \beta=0$. Having made this point and recalling on the other hand (3.7)-(3.8) as $E^{\prime} \ni \varepsilon \rightarrow 0$, we can now pass to the limit in (3.9) using Corollary 3.2 and we get

$$
\left\langle f, u_{0}-\psi_{0}\right\rangle-\iint_{\Omega \times \Delta(A)} b(\mathbb{D} \phi) \cdot(\mathbb{D} \mathbf{u}-\mathbb{D} \phi) d x d \beta \geq 0,
$$

where $\phi$ ranges over $F_{0}^{\infty}$ and hence over $\mathbb{F}_{0}^{1, p}$ too, since the former space is dense in the latter. Therefore, the theorem follows by mere routine (see the proof of [19, Theorem 4.1]).

\section{Concrete homogenization problems for (1.1)}

In this section, we consider a few examples of homogenization problems for (1.1) in a concrete setting (as opposed to the abstract framework of Section 3) and we show how their study leads naturally to the abstract setting of Section 3 and so we may conclude by merely applying Theorem 3.1 .

4.1. Problem I (Periodic setting). We assume here that for each fixed $\xi \in \mathbb{R}^{N}$, the function $(y, z) \rightarrow a(y, z, \xi)$ satisfies the following condition, commonly known as the periodicity hypothesis :

$$
\begin{aligned}
& \text { For each } k \in \mathbb{Z}^{N} \text { and each } \ell \in \mathbb{Z}^{N} \text {, we have } \\
& a(y+k, z+\ell, \xi)=a(y, z, \xi) \text { a.e. in }(y, z) \in \mathbb{R}^{N} \times \mathbb{R}^{N} .
\end{aligned}
$$

One also expresses (4.1) by saying that $a(y, z, \xi)$ (for fixed $\left.\xi \in \mathbb{R}^{N}\right)$ is $Y$ periodic in $y$ and $Z$-periodic in $z$, or simply that $a(y, z, \xi)$ is $Y \times Z$-periodic in $(y, z)$, where $Y=(0,1)^{N}$ and $Z=(0,1)^{N}$ (see Example 2.7).

Our purpose is to study the homogenization of (1.1) under the periodicity hypothesis. This problem was studied in [16] from a different point of view. Here we present an approach which is in keeping with the general pattern of deterministic homogenization theory.

It is clear that the right reiteration $H$-structure for this problem is the periodic $H$-structure $\Sigma=\Sigma_{\mathbb{Z}^{N}} \times \Sigma_{\mathbb{Z}^{N}}$ of Example 2.1. So we have here $\Sigma_{y}=\Sigma_{\mathbb{Z}^{N}}, \Sigma_{z}=\Sigma_{\mathbb{Z}^{N}}$; thus, $A_{y}=\mathcal{C}_{p e r}(Y), A_{z}=\mathcal{C}_{p e r}(Z)$ and $A=\mathcal{C}_{\text {per }}(Y \times Z)$ (see Example 2.7). In this setting, (3.6) takes a rather simple form :

$$
\begin{aligned}
& \mathbf{u}=\left(u_{0}, u_{1}, u_{2}\right) \in \mathbb{F}_{0}^{1, p}: \\
& \int_{\Omega} \int_{Y} \int_{Z} a(y, z, \mathbb{D} \mathbf{u}(x, y, z)) \cdot \mathbb{D} \mathbf{v}(x, y, z) d x d y d z=\left\langle f, v_{0}\right\rangle \\
& \text { for all } \mathbf{v}=\left(v_{0}, v_{1}, v_{2}\right) \in \mathbb{F}_{0}^{1, p},
\end{aligned}
$$


where :

$$
\begin{aligned}
& \mathbb{F}_{0}^{1, p}=W_{0}^{1, p}(\Omega ; \mathbb{R}) \times L^{p}\left(\Omega ; W_{\#}^{1, p}(Y ; \mathbb{R})\right) \times L^{p}\left(\Omega ; L^{p}\left(Y ; W_{\#}^{1, p}(Z ; \mathbb{R})\right)\right), \\
& W_{\#}^{1, p}(Y ; \mathbb{R})=\left\{v \in W_{\text {loc }}^{1, p}\left(\mathbb{R}_{y}^{N} ; \mathbb{R}\right): v Y \text {-periodic, } \int_{Y} v d y=0\right\}, \\
& \mathbb{D} \mathbf{v}=D_{x} v_{0}+D_{y} v_{1}+D_{z} v_{2} \text { for } \mathbf{v}=\left(v_{0}, v_{1}, v_{2}\right) \in \mathbb{F}_{0}^{1, p} .
\end{aligned}
$$

Given an arbitrary real $p>1$, the aim is to show that as $\varepsilon \rightarrow 0$, we have $u_{\varepsilon} \rightarrow u_{0}$ in $W_{0}^{1, p}(\Omega)$-weak and $D_{x_{i}} u_{\varepsilon} \rightarrow D_{x_{i}} u_{0}+D_{y_{i}} u_{1}+D_{z_{i}} u_{2}$ reiteratively in $L^{p}(\Omega)$-weak $\Sigma(1 \leq i \leq N)$, where $u_{\varepsilon}$ is the solution of (1.1) (for fixed $\varepsilon>0$ ) and $\mathbf{u}=\left(u_{0}, u_{1}, u_{2}\right)$ is (uniquely) defined by (4.2). But since $\Sigma$ is $W^{1, p}(\Omega)$-proper (Proposition 2.8), we see by Theorem 3.1 that the whole problem reduces to verifying that (3.1) holds.

Let $1 \leq i \leq N$ and $\psi \in\left(A_{\mathbb{R}}\right)^{N}$ be freely fixed. Let $z \in \mathbb{R}^{N}$ be arbitrarily fixed. In view of (1.2) and part (iii) of (1.4), the function $h: \mathbb{R}^{N} \times \mathbb{R}^{N} \rightarrow \mathbb{R}$ given by $h(y, \xi)=a_{i}(y, z, \xi) \quad\left(y, \xi \in \mathbb{R}^{N}\right)$ has the Caratheodory property. Hence, a classical result (see, e.g., [11, p.75]) reveals that if $u$ is any measurable function from $\mathbb{R}^{N}$ into $\mathbb{R}^{N}$, then the function $y \rightarrow h(y, u(y))$ is measurable from $\mathbb{R}^{N}$ into $\mathbb{R}$. Choosing in particular $u(y)=\psi(y, z) \quad\left(y \in \mathbb{R}^{N}\right)$, we see that the function $y \rightarrow a_{i}(y, z, \psi(y, z))$ is measurable from $\mathbb{R}^{N}$ into $\mathbb{R}$, and that for any arbitrary $z$ in $\mathbb{R}^{N}$. On the other hand, by a routine calculation using [part (ii) of] (1.2) and [part (iii) of] (1.4) one can easily show that the function $z \rightarrow a_{i}(y, z, \psi(y, z))$ (for fixed $y$ ) is continuous on $\mathbb{R}^{N}$. Taking account of (4.1), we deduce that, a.e. in $y \in \mathbb{R}^{N}$, the function $z \rightarrow a_{i}(y, z, \psi(y, z))$ of $\mathbb{R}^{N}$ into $\mathbb{R}$, denoted below by $a_{i}(y, \cdot, \psi(y, \cdot))$, lies in $\mathcal{C}_{p e r}(Z)$. Hence it follows by Lemma 2.1 that the function $y \rightarrow a_{i}(y, \cdot, \psi(y, \cdot))$, denoted by $a_{i}(\cdot, \cdot, \psi)$, is measurable from $\mathbb{R}^{N}$ into $\mathcal{C}_{\text {per }}(Z)$. From this we deduce using (1.3), part (iii) of (1.4), and (4.1), that $a_{i}(\cdot, \cdot, \psi)$ lies in $L_{p e r}^{\infty}\left(Y ; \mathcal{C}_{\text {per }}(Z)\right)$. But $L_{\text {per }}^{\infty}\left(Y ; \mathcal{C}_{\text {per }}(Z)\right) \subset L_{\text {per }}^{p^{\prime}}\left(Y ; \mathcal{C}_{\text {per }}(Z)\right)$. Hence $(3.1)$ follows by the facts that $A=\mathcal{C}_{\text {per }}(Y \times Z)$ is dense in $L_{\text {per }}^{p^{\prime}}\left(Y ; \mathcal{C}_{\text {per }}(Z)\right)$ and the latter space is continuously embedded in $\Xi^{p^{\prime}}\left(\mathbb{R}_{y}^{N} ; \mathcal{B}\left(\mathbb{R}_{z}^{N}\right)\right)$.

4.2. Problem II. We study here the homogenization of (1.1) under the structure hypothesis

$$
a_{i}(\cdot, \cdot, \xi) \in \mathcal{B}_{\infty}\left(\mathbb{R}_{z}^{N} ; \mathcal{C}_{p e r}(Y)\right) \text { for any } \xi \in \mathbb{R}^{N}(1 \leq i \leq N),
$$

where $\mathcal{B}_{\infty}\left(\mathbb{R}_{z}^{N} ; \mathcal{C}_{\text {per }}(Y)\right)$ denotes the space of continuous functions $\psi$ : $\mathbb{R}_{z}^{N} \rightarrow \mathcal{C}_{\text {per }}(Y)$ such that $\psi(z)=\psi(\cdot, z)$ has a limit in $\mathcal{B}\left(\mathbb{R}_{y}^{N}\right)$ when $|z| \rightarrow \infty$. 
Proposition 4.1. Let (4.3) be satisfied. Then (3.1) holds with $\Sigma=$ $\Sigma_{\mathbb{Z}^{N}} \times \Sigma_{\infty}$ (i.e., with $\Sigma_{y}=\Sigma_{\mathbb{Z}^{N}}$ and $\Sigma_{z}=\Sigma_{\infty}$ ) as in Example 2.10, so that the conclusion of Theorem 3.1 holds for $p=2$.

Proof. First of all, we note that $\mathcal{B}_{\infty}\left(\mathbb{R}_{z}^{N} ; \mathcal{C}_{\text {per }}(Y)\right)$ is exactly the image of the reiteration $H$-structure $\Sigma=\Sigma_{\mathbb{Z}^{N}} \times \Sigma_{\infty}$ (Example 2.4) and further the latter is $W^{1,2}(\Omega)$-proper. Therefore, the proof is complete once we have established that

$$
a_{i}(\cdot, \cdot, \psi) \in A=\mathcal{B}_{\infty}\left(\mathbb{R}_{z}^{N} ; \mathcal{C}_{p e r}(Y)\right) \text { for all } \psi \in\left(A_{\mathbb{R}}\right)^{N}(1 \leq i \leq N) .
$$

To do this, let $1 \leq i \leq N$ and $\psi \in\left(A_{\mathbb{R}}\right)^{N}$ be freely fixed. Let $K$ be a compact set in $\mathbb{R}^{N}$ such that $\psi(y, z) \in K$ for all $(y, z) \in \mathbb{R}^{N} \times \mathbb{R}^{N}$. According to (4.3), we may view $a_{i}$ as a function $\xi \rightarrow a_{i}(\cdot, \cdot, \xi)$ of $\mathbb{R}^{N}$ into $A_{\mathbb{R}}$, which function lies in $\mathcal{C}\left(\mathbb{R}^{N} ; A_{\mathbb{R}}\right)$ (use part (iii) of (1.4)). Still calling $a_{i}$ the restriction of the latter function to $K$, we have therefore $a_{i} \in \mathcal{C}\left(K ; A_{\mathbb{R}}\right)$. Recalling that $\mathcal{C}_{\mathbb{R}}(K) \otimes A_{\mathbb{R}}$ is dense in $\mathcal{C}\left(K ; A_{\mathbb{R}}\right)$ (see, e.g., [6, p.46]), we see that we may consider a sequence $\left(q_{n}\right)_{n \geq 1}$ in $\mathcal{C}_{\mathbb{R}}(K) \otimes A_{\mathbb{R}}$ such that

$$
\sup _{(y, z, \xi) \in \mathbb{R}^{N} \times \mathbb{R}^{N} \times K}\left|q_{n}(y, z, \xi)-a_{i}(y, z, \xi)\right| \rightarrow 0 \text { as } n \rightarrow \infty .
$$

Hence $q_{n}(\cdot, \cdot, \psi) \rightarrow a_{i}(\cdot, \cdot, \psi)$ in $\mathcal{B}\left(\mathbb{R}_{y}^{N} \times \mathbb{R}_{z}^{N}\right)$ as $n \rightarrow \infty$. Thus, (4.4) is proved if we can check that each $q_{n}(\cdot, \cdot,, \psi)$ lies in $A$. However, it is enough to verify that we have $q(\cdot, \cdot, \psi) \in A$ for any function $q: \mathbb{R}_{y}^{N} \times \mathbb{R}_{z}^{N} \times \mathbb{R}_{\xi}^{N} \rightarrow \mathbb{R}$ of the form

$$
q(y, z, \xi)=\chi(\xi) \phi(y, z)\left(y, z, \xi \in \mathbb{R}^{N}\right) \text { with } \chi \in \mathcal{C}(K ; \mathbb{R}) \text { and } \phi \in A_{\mathbb{R}} .
$$

Given one such $q$, by the Stone-Weierstrass theorem we may consider a sequence $\left(f_{n}\right)$ of polynomials in $\xi=\left(\xi_{1}, \cdots, \xi_{N}\right) \in K$ such that $f_{n} \rightarrow \chi$ in $\mathcal{C}(K)$ as $n \rightarrow \infty$, hence $f_{n}(\psi) \rightarrow \chi(\psi)$ in $\mathcal{B}\left(\mathbb{R}_{y}^{N} \times \mathbb{R}_{z}^{N}\right)$ as $n \rightarrow \infty$, where $f_{n}(\psi)$ stands for $f_{n} \circ \psi$ (usual composition) and $\chi(\psi)$ stands for $\chi \circ \psi$. We deduce that $\chi(\psi)$ lies in $A_{\mathbb{R}}$, since the same is true of each $f_{n}(\psi)\left(A_{\mathbb{R}}\right.$ being an algebra). The proposition follows thereby.

4.3. Problem III. We investigate here the limiting behaviour, as $\varepsilon \rightarrow 0$, of $u_{\varepsilon}$ (defined for each $\varepsilon>0$ by (1.1)) under the structure hypothesis

$$
a_{i}(\cdot, \cdot, \xi) \in \mathcal{B}_{\infty}\left(\mathbb{R}_{z}^{N} ; A P\left(\mathbb{R}_{y}^{N}\right)\right) \text { for any } \xi \in \mathbb{R}^{N}(1 \leq i \leq N)
$$

where $A P\left(\mathbb{R}_{y}^{N}\right)$ is the space of almost periodic continuous complex functions on $\mathbb{R}_{y}^{N}[5,12,13]$. 
Proposition 4.2. Let (4.5) be satisfied. There is a countable subgroup $\mathcal{R}_{y}$ of $\mathbb{R}_{y}^{N}$ such that (3.1) holds with $\Sigma=\Sigma_{\mathcal{R}_{y}} \times \Sigma_{\infty}$ as in Example 2.10, so that the conclusion of Theorem 3.1 holds for $p=2$.

Proof. As shown in [20, Corollary 4.1], there exists a countable subgroup, $\mathcal{R}_{y}$, of $\mathbb{R}_{y}^{N}$ such that $a_{i}(\cdot, \cdot, \xi) \in \mathcal{B}_{\infty}\left(\mathbb{R}_{z}^{N} ; A P_{\mathcal{R}_{y}}\left(\mathbb{R}_{y}^{N}\right)\right)$ for all $\xi \in \mathbb{R}^{N}$ and all $1 \leq i \leq N$, where $A P_{\mathcal{R}_{y}}\left(\mathbb{R}_{y}^{N}\right)$ denotes the space of those $u \in A P\left(\mathbb{R}_{y}^{N}\right)$ such that $M\left(\bar{\gamma}_{k} u\right)=0$ for all $k \in \mathbb{R}^{N} \backslash \mathcal{R}_{y} \quad\left(\gamma_{k}\right.$ is as in Example 2.1 and $M$ is the mean value on $\mathbb{R}^{N}$ for the action $\left.\mathcal{H}\right)$. But then the space $A=\mathcal{B}_{\infty}\left(\mathbb{R}_{z}^{N} ; A P_{\mathcal{R}_{y}}\left(\mathbb{R}_{y}^{N}\right)\right)$ is nothing else but the image of the $W^{1,2}(\Omega)$ proper $H$-structure $\Sigma=\Sigma_{\mathcal{R}_{y}} \times \Sigma_{\infty}$. Hence, we are through with the proof if we can show that $a_{i}(\cdot, \cdot, \psi) \in A$ for all $\psi \in\left(A_{\mathbb{R}}\right)^{N}$ and all $1 \leq i \leq N$. But this follows by repeating word for word the proof of (4.4).

4.4. Problem IV. The case to be examined in the present subsection states as above except that in place of (4.5) we have here the almost periodicity hypothesis

$$
a_{i}(\cdot, \cdot, \xi) \in A P\left(\mathbb{R}_{y}^{N} \times \mathbb{R}_{z}^{N}\right) \text { for all } \xi \in \mathbb{R}^{N}(1 \leq i \leq N)
$$

Proposition 4.3. Under assumption (4.6), condition (3.1) is fulfilled with $\Sigma=\Sigma_{\mathcal{R}_{y}} \times \Sigma_{\mathcal{R}_{z}}$ (Example 2.2), where $\mathcal{R}_{y}$ (resp. $\mathcal{R}_{z}$ ) is a suitable countable subgroup of $\mathbb{R}_{y}^{N}$ (resp. $\mathbb{R}_{z}^{N}$ ). Since $\Sigma$ is $W^{1,2}(\Omega)$-proper (Proposition 2.9), the conclusion of Theorem 3.1 holds for $p=2$.

Proof. Appeal to [20, Corollary 4.1], once again, yields some countable subgroup $R$ of $\mathbb{R}^{2 N}=\mathbb{R}^{N} \times \mathbb{R}^{N}$ such that $a_{i}(\cdot, \cdot, \xi) \in A P_{R}\left(\mathbb{R}^{2 N}\right)$ for all $\xi \in \mathbb{R}^{N}$ and all $1 \leq i \leq N$. Letting $\mathcal{R}_{y}=p r_{y}(R)$ and $\mathcal{R}_{z}=p r_{z}(R)$, where $p r_{y}$ (resp. $p r_{z}$ ) denotes the natural projection of $\mathbb{R}^{2 N}=\mathbb{R}_{y}^{N} \times \mathbb{R}_{z}^{N}$ onto $\mathbb{R}_{y}^{N}$ (resp. $\mathbb{R}_{z}^{N}$ ), we deduce

$$
a_{i}(\cdot, \cdot, \xi) \in A=A P_{\mathcal{R}}\left(\mathbb{R}_{y}^{N} \times \mathbb{R}_{z}^{N}\right) \text { for all } \xi \in \mathbb{R}^{N}(1 \leq i \leq N)
$$

where $\mathcal{R}=\mathcal{R}_{y} \times \mathcal{R}_{z}$ (a countable subgroup of $\mathbb{R}^{N} \times \mathbb{R}^{N}$ ). But then $A P_{\mathcal{R}}\left(\mathbb{R}_{y}^{N} \times \mathbb{R}_{z}^{N}\right)$ is precisely the image of $\Sigma=\Sigma_{\mathcal{R}_{y}} \times \Sigma_{\mathcal{R}_{z}}$ (see [18, Examples 3.3 and 3.6]), and the latter is $W^{1,2}(\Omega)$-proper. Therefore the proposition is proved if we can show that $a_{i}(\cdot, \cdot, \psi) \in A=A P_{\mathcal{R}}\left(\mathbb{R}_{y}^{N} \times \mathbb{R}_{z}^{N}\right)$ for all $\psi \in\left(A_{\mathbb{R}}\right)^{N}$ and all $1 \leq i \leq N$. But the proof of this is a verbatim copy of that of (4.4) and therefore is not worth repeating.

4.5. Problem V. Let $\mathcal{B}_{\infty, \mathbb{Z}^{N}}\left(\mathbb{R}_{y}^{N}\right)$ denote the closure in $\mathcal{B}\left(\mathbb{R}_{y}^{N}\right)$ of the space of all finite sums $\sum_{\text {finite }} \varphi_{i} u_{i}$ with $\varphi_{i} \in \mathcal{B}_{\infty}\left(\mathbb{R}_{y}^{N}\right), u_{i} \in \mathcal{C}_{\text {per }}(Y)$, where 
$\mathcal{B}_{\infty}\left(\mathbb{R}_{y}^{N}\right)$ is the space of those continuous complex functions on $\mathbb{R}_{y}^{N}$ that converge at infinity.

We intend to study the homogenization of (1.1) under the structure hypothesis

$$
a_{i}(\cdot, \cdot, \xi) \in \mathcal{B}_{\infty}\left(\mathbb{R}_{z}^{N} ; \mathcal{B}_{\infty, \mathbb{Z}^{N}}\left(\mathbb{R}_{y}^{N}\right)\right) \text { for all } \xi \in \mathbb{R}^{N}(1 \leq i \leq N) .
$$

Proposition 4.4. Under hypothesis (4.7), we have (3.1) with $\Sigma=$ $\Sigma_{\infty, \mathbb{Z}^{N}} \times \Sigma_{\infty}$. Furtheremore, the $H$-structure $\Sigma$ is $W^{1,2}(\Omega)$-proper, so that the conclusion of Theorem 3.1 holds for $p=2$.

Proof. It is worth recalling that $\mathcal{B}_{\infty, \mathbb{Z}^{N}}\left(\mathbb{R}_{y}^{N}\right)$ is an $H$-algebra on $\mathbb{R}_{y}^{N}$ for $\mathcal{H}$, and $\Sigma_{\infty, \mathbb{Z}^{N}}$ denotes the $H$-structure of which $\mathcal{B}_{\infty, \mathbb{Z}^{N}}\left(\mathbb{R}_{y}^{N}\right)$ is the image (see [18, Example 3.5]). Now, there is no difficulty in showing that $\mathcal{B}_{\infty}\left(\mathbb{R}_{z}^{N}\right) \otimes \mathcal{B}_{\infty, \mathbb{Z}^{N}}\left(\mathbb{R}_{y}^{N}\right)$ is dense in $\mathcal{B}_{\infty}\left(\mathbb{R}_{z}^{N} ; \mathcal{B}_{\infty, \mathbb{Z}^{N}}\left(\mathbb{R}_{y}^{N}\right)\right)$. Hence, recalling that $\Sigma_{\infty}$ is the $H$-structure on $\mathbb{R}_{z}^{N}$ (for $\mathcal{H}^{\prime}$ ) of which $\mathcal{B}_{\infty}\left(\mathbb{R}_{z}^{N}\right)$ is the image (see [18, Example 3.4]), it follows by [18, Proposition 3.2] that the space $A=\mathcal{B}_{\infty}\left(\mathbb{R}_{z}^{N} ; \mathcal{B}_{\infty, \mathbb{Z}^{N}}\left(\mathbb{R}_{y}^{N}\right)\right)$ is exactly the image of the $H$-structure $\Sigma=\Sigma_{\infty, \mathbb{Z}^{N}} \times \Sigma_{\infty}$ on $\mathbb{R}_{y}^{N} \times \mathbb{R}_{z}^{N}$ (for $\mathcal{H}^{*}$ ). On the other hand, the latter is $W^{1,2}(\Omega)$-proper, as is immediate by Example 2.11 and use of the fact that $\Sigma_{\infty}=\Sigma_{\infty, \mathcal{R}_{z}=\{\omega\}}$ ( $\omega$ the origin in $\left.\mathbb{R}_{z}^{N}\right)$. Therefore, the proof is complete once we have verified that $a_{i}(\cdot, \cdot, \psi) \in A$ for all $\psi \in\left(A_{\mathbb{R}}\right)^{N}$ and all $1 \leq i \leq N$. But this follows by repeating word for word the proof of (4.4).

Corollary 4.1. Suppose we have $a_{i}(\cdot, \cdot, \xi) \in \mathcal{B}_{\infty}\left(\mathbb{R}_{z}^{N} ; \mathcal{B}_{\infty}\left(\mathbb{R}_{y}^{N}\right)\right)$ for all $\xi \in \mathbb{R}^{N}$ and all $1 \leq i \leq N$. Then, the conclusion of Proposition 4.4 stands.

Proof. Indeed, we have $\mathcal{B}_{\infty}\left(\mathbb{R}_{z}^{N} ; \mathcal{B}_{\infty}\left(\mathbb{R}_{y}^{N}\right)\right) \subset \mathcal{B}_{\infty}\left(\mathbb{R}_{z}^{N} ; \mathcal{B}_{\infty, \mathbb{Z}^{N}}\left(\mathbb{R}_{y}^{N}\right)\right)$, hence the desired result follows by the preceding proposition.

4.6. Problem VI. The problem to be worked out here states as in Subsection 4.5 except that (4.7) is replaced by the structure hypothesis

$$
a_{i}(\cdot, \cdot, \xi) \in \mathcal{C}_{\text {per }}\left(Z ; \mathcal{B}_{\infty, \mathbb{Z}^{N}}\left(\mathbb{R}_{y}^{N}\right)\right), \text { for all } \xi \in \mathbb{R}^{N}(1 \leq i \leq N)
$$

where $\mathcal{C}_{\text {per }}\left(Z ; \mathcal{B}_{\infty, \mathbb{Z}^{N}}\left(\mathbb{R}_{y}^{N}\right)\right)$ is the space of $Z$-periodic continuous functions of $\mathbb{R}_{z}^{N}$ into $\mathcal{B}_{\infty, \mathbb{Z}^{N}}\left(\mathbb{R}_{y}^{N}\right)$.

Proposition 4.5. Under hypothesis (4.8), we have (3.1) with $\Sigma=$ $\Sigma_{\infty, \mathbb{Z}^{N}} \times \Sigma_{\mathbb{Z}^{N}}$. Furtheremore, the $H$-structure $\Sigma$ is $W^{1, p}(\Omega)$-proper, so that the conclusion of Theorem 3.1 holds.

Proof. The space $A=\mathcal{C}_{\text {per }}\left(Z ; \mathcal{B}_{\infty, \mathbb{Z}^{N}}\left(\mathbb{R}_{y}^{N}\right)\right)$ coincides with the closure of $\mathcal{B}_{\infty, \mathbb{Z}^{N}}\left(\mathbb{R}_{y}^{N}\right) \otimes \mathcal{C}_{\text {per }}(Z)$ in $\mathcal{B}\left(\mathbb{R}_{y}^{N} \times \mathbb{R}_{z}^{N}\right)$. Hence it follows by $[18$, Proposition 
3.2] that $A$ is nothing but the image of the $H$-structure $\Sigma=\Sigma_{\infty, \mathbb{Z}^{N}} \times \Sigma_{\mathbb{Z}^{N}}$. On the other hand, by a procedure similar to that followed in Example 2.9 it can be shown that $\Sigma$ is $W^{1, p}(\Omega)$-proper. Therefore, the proposition is proved if we can check that $a_{i}(\cdot, \cdot, \psi)$ lies in $A$ for all $\psi \in\left(A_{\mathbb{R}}\right)^{N}$ and all $1 \leq i \leq N$ which can be obtained by repeating the proof of (4.4).

4.7. Problem VII. We assume that the following condition is satisfied :

For each bounded set $\Lambda \subset \mathbb{R}^{N}$ and each real $\eta>0$, there is some $\rho>0$ such that $|a(y-t, z-\sigma, \xi)-a(y, z, \xi)| \leq \eta$ for all $(z, \xi) \in \mathbb{R}^{N} \times \Lambda$ and for almost all $y \in \mathbb{R}^{N}$ provided $|t|+|\sigma| \leq \rho$

and we want to investigate the homogenization of (1.1) under the structure hypothesis

$$
a_{i}(\cdot, \cdot, \xi) \in \mathcal{B}_{\infty}\left(\mathbb{R}_{z}^{N} ; L_{\text {per }}^{2}(Y)\right) \text { for all } \xi \in \mathbb{R}^{N}(1 \leq i \leq N)
$$

which generalizes (4.3).

For this purpose, let us introduce a suitable function space generalizing the usual amalgam of $L^{p}$ and $\ell^{\infty}$ on $\mathbb{R}^{N}$ (see [10]). Let $1 \leq p<$ $\infty$. We define $\left(L^{p}, \ell^{\infty}\right)\left(\mathbb{R}_{y}^{N} ; \mathcal{B}\left(\mathbb{R}_{z}^{N}\right)\right)$ to be the space of functions $u \in$ $L_{l o c}^{p}\left(\mathbb{R}_{y}^{N} ; \mathcal{B}\left(\mathbb{R}_{z}^{N}\right)\right)$ such that

$$
\|u\|_{p, \infty}=\sup _{k \in \mathbb{Z}^{N}}\left(\int_{k+Y}\|u(y, \cdot)\|_{\infty}^{p} d y\right)^{\frac{1}{p}}<\infty .
$$

This is a vector space over $\mathbb{C}$ and further $\|\cdot\|_{p, \infty}$ is a norm under which $\left(L^{p}, \ell^{\infty}\right)\left(\mathbb{R}_{y}^{N} ; \mathcal{B}\left(\mathbb{R}_{z}^{N}\right)\right)$ is a Banach space. One fundamental result is that $\left(L^{p}, \ell^{\infty}\right)\left(\mathbb{R}_{y}^{N} ; \mathcal{B}\left(\mathbb{R}_{z}^{N}\right)\right)$ is continuously embedded in $\Xi^{p}\left(\mathbb{R}_{y}^{N} ; \mathcal{B}\left(\mathbb{R}_{z}^{N}\right)\right.$ ) (this is immediate by $[20$, inequality $(4.1)])$. Let us turn now to the proof of

Proposition 4.6. Let (4.9)-(4.10) hold. Then (3.1) is satisfied with $p=2$ and $\Sigma=\Sigma_{\mathbb{Z}^{N}} \times \Sigma_{\infty}\left(W^{1,2}(\Omega)\right.$-proper reiteration $H$-stucture on $\mathbb{R}^{N} \times \mathbb{R}^{N}$ for $\left.\mathcal{H}^{*}\right)$, so that the conclusion of Theorem 3.1 holds for $p=2$.

Proof. Let $\left(\theta_{n}\right)_{n \geq 1}$ be a mollifier on $\mathbb{R}_{y}^{N}$, i.e., $\left(\theta_{n}\right)_{n \geq 1} \subset C_{0}^{\infty}\left(\mathbb{R}_{y}^{N}\right)$ with $\theta_{n} \geq 0, \int \theta_{n}(y) d y=1, \theta_{n}$ has support in $\varepsilon_{n} \bar{B}_{N}$, where $\bar{B}_{N}$ is the closed unit ball in $\mathbb{R}_{y}^{N}$ and $0<\varepsilon_{n} \leq 1$ with $\varepsilon_{n} \rightarrow 0$ as $n \rightarrow \infty$. Let $n$ be freely fixed. For any arbitrary $1 \leq i \leq N$, let

$$
q_{n}^{i}(y, z, \xi)=\int \theta_{n}(t) a_{i}(y-t, z, \xi) d t \quad\left(y, z, \xi \in \mathbb{R}^{N}\right)
$$


which gives a function $(y, z, \xi) \rightarrow q_{n}^{i}(y, z, \xi)$ of $\mathbb{R}^{N} \times \mathbb{R}^{N} \times \mathbb{R}^{N}$ into $\mathbb{R}$ with

$$
q_{n}^{i}(\cdot, \cdot, \xi) \in \mathcal{C}\left(\mathbb{R}_{z}^{N} ; \mathcal{C}_{p e r}(Y)\right) \text { for any } \xi \in \mathbb{R}^{N},
$$

as it follows by (4.9). Furthermore, on letting $q_{n}=\left(q_{n}^{i}\right)_{1 \leq i \leq N}$ and recalling (1.3)-(1.4), we have for all $z \in \mathbb{R}^{N}$ and almost all $y \in \mathbb{R}^{N}, q_{n}(y, z, \omega)=0$ and

$$
\left|q_{n}\left(y, z, \xi_{1}\right)-q_{n}\left(y, z, \xi_{2}\right)\right| \leq c_{1}\left(1+\left|\xi_{1}\right|+\left|\xi_{2}\right|\right)^{p-1-\alpha_{1}}\left|\xi_{1}-\xi_{2}\right|^{\alpha_{1}},
$$

where $\xi_{1}$ and $\xi_{2}$ are arbitrary.

The next point is to show that $q_{n}^{i}(\cdot, z, \xi)$ (where $\xi$ is fixed) has a limit in $\mathcal{B}\left(\mathbb{R}_{y}^{N}\right)$ when $|z| \rightarrow \infty$. To this end, let us introduce the mapping $\xi \rightarrow a_{i}^{*}(\cdot, \xi)$ of $\mathbb{R}^{N}$ into $L_{\text {per }}^{2}(Y)$ such that $a_{i}(\cdot, z, \xi) \rightarrow a_{i}^{*}(\cdot, \xi)$ in $L^{2}(Y)$ as $|z| \rightarrow \infty$ (see (4.10)), where $\xi$ is arbitrarily fixed. Put

$$
p_{n}^{i}(y, \xi)=\int \theta_{n}(t) a_{i}^{*}(y-t, \xi) d t \quad\left(y, \xi \in \mathbb{R}^{N}\right) .
$$

This defines a function $(y, \xi) \rightarrow p_{n}^{i}(y, \xi)$ of $\mathbb{R}^{N} \times \mathbb{R}^{N}$ into $\mathbb{R}$ with $p_{n}^{i}(\cdot, \xi) \in$ $\mathcal{C}_{\text {per }}(Y)$ for each fixed $\xi$. Let us show that $q_{n}^{i}(\cdot, z, \xi) \rightarrow p_{n}^{i}(\cdot, \xi)$ as $|z| \rightarrow \infty$.

To do this, we start from

$$
q_{n}^{i}(y, z, \xi)-p_{n}^{i}(y, \xi)=\int \theta_{n}(t)\left[a_{i}(y-t, z, \xi)-a_{i}^{*}(y-t, \xi)\right] d t .
$$

We apply Holder's inequality to get

$$
\left|q_{n}^{i}(y, z, \xi)-p_{n}^{i}(y, \xi)\right| \leq c_{n}\left(\int_{B_{N}}\left|a_{i}(y-t, z, \xi)-a_{i}^{*}(y-t, \xi)\right|^{2} d t\right)^{\frac{1}{2}},
$$

where $c_{n}=\left\|\theta_{n}\right\|_{\infty}^{\frac{1}{2}}$. Now, note that as $k$ ranges over $\mathbb{Z}^{N}$, the sets $k+\bar{Y}$ form a covering of $\mathbb{R}^{N}$. Thus, we may consider a finite set $S \subset \mathbb{Z}^{N}$ such that $B_{N}$ is contained in the union $\bigcup_{k \in S}(k+\bar{Y})$. Then $y-B_{N}$ is contained in $\bigcup_{k \in S}(k+y+\bar{Y})$ (where $y$ is freely fixed in $\mathbb{R}^{N}$ ). Hence, by the change of variable $\sigma=y-t$ in the preceding integral and use of the $Y$-periodicity we arrive at

$$
\left|q_{n}^{i}(y, z, \xi)-p_{n}^{i}(y, \xi)\right| \leq c_{n}|S|^{\frac{1}{2}}\left(\int_{Y}\left|a_{i}(\sigma, z, \xi)-a_{i}^{*}(\sigma, \xi)\right|^{2} d \sigma\right)^{\frac{1}{2}},
$$


where $|S|$ denotes the cardinality of $S$. This being so, let $\eta>0$. Let $\rho>0$ be such that

$$
\left(\int_{Y}\left|a_{i}(\sigma, z, \xi)-a_{i}^{*}(\sigma, \xi)\right|^{2} d \sigma\right)^{\frac{1}{2}} \leq \frac{\eta}{c_{n}|S|^{\frac{1}{2}}}
$$

for any $z \in \mathbb{R}^{N}$ verifying $|z| \geq \rho$. Then, clearly

$$
\sup _{y \in \mathbb{R}^{N}}\left|q_{n}^{i}(y, z, \xi)-p_{n}^{i}(y, \xi)\right| \leq \eta \quad\left(z \in \mathbb{R}^{N},|z| \geq \rho\right) .
$$

Therefore

$$
q_{n}^{i}(\cdot, \cdot, \xi) \in \mathcal{B}_{\infty}\left(\mathbb{R}_{z}^{N} ; \mathcal{C}_{p e r}(Y)\right), \text { for all } \xi \in \mathbb{R}^{N}(1 \leq i \leq N)
$$

where the integer $n \geq 1$ is arbitrary. So we are justified in carrying over to the present situation the line of argument leading to (4.4). This yields

$$
q_{n}^{i}(\cdot, \cdot, \psi) \in A=\mathcal{B}_{\infty}\left(\mathbb{R}_{z}^{N} ; \mathcal{C}_{p e r}(Y)\right) \text { for all } \psi \in\left(A_{\mathbb{R}}\right)^{N}(1 \leq i \leq N) .
$$

Let us next show the following assertion

Let $\psi \in\left(A_{\mathbb{R}}\right)^{N}$ and $1 \leq i \leq N$ be fixed. To each $\eta>0$ there is assigned some integer $\nu \geq 1$ such that $\left\|q_{n}^{i}(\cdot, \cdot, \psi)-a_{i}(\cdot, \cdot, \psi)\right\|_{2, \infty} \leq \eta$ for any integer $n \geq \nu$.

First, by Holder's inequality we have

$$
\begin{aligned}
& \left|q_{n}^{i}(y, z, \psi(y, z))-a_{i}(y, z, \psi(y, z))\right|^{2} \\
& \leq \int \theta_{n}(t)\left|a_{i}(y-t, z, \psi(y, z))-a_{i}(y, z, \psi(y, z))\right|^{2} d t .
\end{aligned}
$$

On the other hand, given $\eta>0$, let $\rho>0$ be such that $\left|a_{i}(y-t, z, \xi)-a_{i}(y, z, \xi)\right| \leq \eta$ for almost all $y \in \mathbb{R}^{N}$ and for all $(z, \xi) \in$ $\mathbb{R}^{N} \times \Lambda$ provided $|t| \leq \rho$, where $\Lambda$ is a compact set in $\mathbb{R}^{N}$ containing the range of $\psi$. Finally, let $\nu$ be a positive integer such that $\varepsilon_{n} \leq \rho$ for any $n \geq \nu$. Then, recalling that integration above is actually taken over $\varepsilon_{n} B_{N}$, one quickly arrives at

$$
\left|q_{n}^{i}(y, z, \psi(y, z))-a_{i}(y, z, \psi(y, z))\right|^{2} \leq \eta^{2}
$$

for any integer $n \geq \nu$, for all $z \in \mathbb{R}^{N}$ and for almost all $y \in \mathbb{R}^{N}$. Hence (4.2) follows in an obvious manner. Combining this with (4.11) and recalling that $\left(L^{2}, \ell^{\infty}\right)\left(\mathbb{R}_{y}^{N} ; \mathcal{B}\left(\mathbb{R}_{z}^{N}\right)\right)$ is continuously embedded in $\Xi^{2}\left(\mathbb{R}_{y}^{N} ; \mathcal{B}\left(\mathbb{R}_{z}^{N}\right)\right)$, we obtain (3.1) with $p=2$ and $\Sigma=\Sigma_{\mathbb{Z}^{N}} \times \Sigma_{\infty}$, which completes the proof. $\square$ 
Remark 4.1. It can be proved by a similar approach that Proposition 4.2 remains valid if in (4.5) $A P\left(\mathbb{R}_{y}^{N}\right)$ is substituted by $L_{A P}^{2}\left(\mathbb{R}_{y}^{N}\right)$ (the space of those functions in $L_{l o c}^{2}\left(\mathbb{R}_{y}^{N}\right)$ that are almost periodic in the Stepanoff sense $[10,17])$ provided $(4.9)$ is satisfied.

\section{References}

[1] R. Adams, Sobolev Spaces, Academic Press, New York, 1975.

[2] M. Baia and I. Fonseca, The limit behavior of a family of variational multiscale problems, Scientific Report 2005, Carnegie Mellon, Dep. Math. Sciences, 05-CNA-017.

[3] M. Baia and I. Fonseca, $\Gamma$-convergence of functionals with periodic integrands via 2-scale convergence, Scientific Report 2005, Carnegie Mellon, Dep. Math. Sciences, 05-CNA-010.

[4] A. Bensoussan, J.L. Lions and G. Papanicolaou, Asymptotic Analysis for Periodic Structures, North-Holland, Amsterdam, 1978.

[5] A.S. Besicovitch, Almost Periodic Functions, Dover Publications, Cambridge, 1954.

[6] N. Bourbaki, Intégration, Hermann, Paris, 1966, Chapters 1-4.

[7] N. Bourbaki, Intégration, Hermann, Paris, 1967, Chapter 5.

[8] M. Briane and G. Allaire, Multiscale convergence and reiterated homogenization, Proc. Roy. Soc. Edinburgh, Sect.A, 126 (1996) 297342.

[9] R.E. Edwards, Functional Analysis, Holt-Rinehart-Winston, New York, 1965.

[10] J.J.F. Fournier and J. Stewart, Amalgams of $L^{p}$ and $\ell^{q}$, Bull. Amer. Math. Soc., 13 (1985), 1-21.

[11] S. Fučik and A. Kufner, Nonlinear Differential Equations, Elsevier Scientific Publishing Company, Amsterdam-Oxford-New York, 1980.

[12] A. Guichardet, Analyse Harmonique Commutative, Dunod, Paris, 1968 .

[13] R. Larsen, Banach Algebras, Marcel Dekker, New York, 1973.

[14] J.L. Lions, D. Lukkassen, L.E. Persson and P. Wall, Reiterated homogenization of monotone operators, C.R.Acad. Sci. Paris, Sér. I Math., 330 (2000), 675-680.

[15] J.L. Lions, D. Lukkassen, L.E. Persson and P. Wall, Reiterated homogenization of nonlinear monotone operators, Chin. Ann. of Math., 22 (2001), 1-12. 
[16] D. Lukkassen, G. Nguetseng and P. Wall, Two-scale convergence, Int. J. Pure Appl. Math., 2 (2002), 35-86.

[17] G. Nguetseng, Almost periodic homogenization : asymptotic analysis of a second order elliptic equation, Preprint.

[18] G. Nguetseng, Homogenization structures and Applications I, Z. Anal. Anwend., 22 (2003) 73-107.

[19] G. Nguetseng, H. Nnang, Homogenization of nonlinear monotone operators beyond the periodic setting, Electr. J. Diff. Equations, 36, (2003) 1-24.

[20] G. Nguetseng, J.L. Woukeng, Deterministic homogenization of parabolic monotone operators with time dependent coefficients, Electr. J. Diff. Equations, 82 (2004) 1-23.

Narvik University College

P.O. Box 385, N-8505 Narvik

Norway

and

Norut Narvik

P.O. Box 250, N-8504 Narvik

Norway

(E-mail : dl@hin.no)

Department of Mathematics

University of Yaounde I

P.O. Box 812 Yaounde

Cameroon

(E-mail : nguetsengg@yahoo.fr)

University of Yaounde I

Ecole Normale Supérieure

P.O. Box 47 Yaounde

Cameroon

(E-mail : hnnang@yahoo.fr)

Department pf Mathematics

Luleå Technological University

S-97187 Luleå

Sweden (E-mail : wall@sm.luth.se)

(Received : January 2008) 


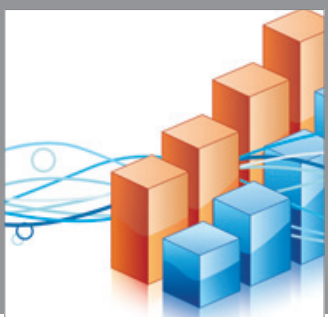

Advances in

Operations Research

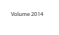

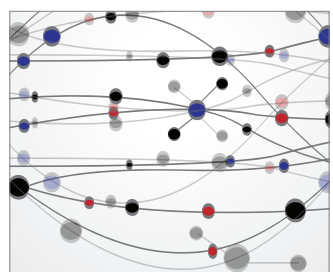

\section{The Scientific} World Journal
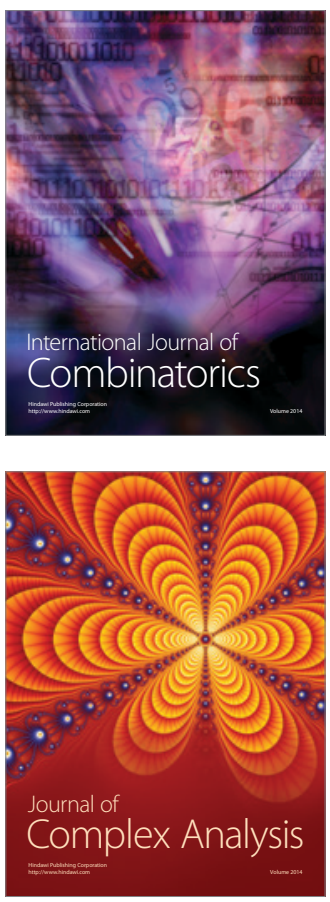

International Journal of

Mathematics and

Mathematical

Sciences
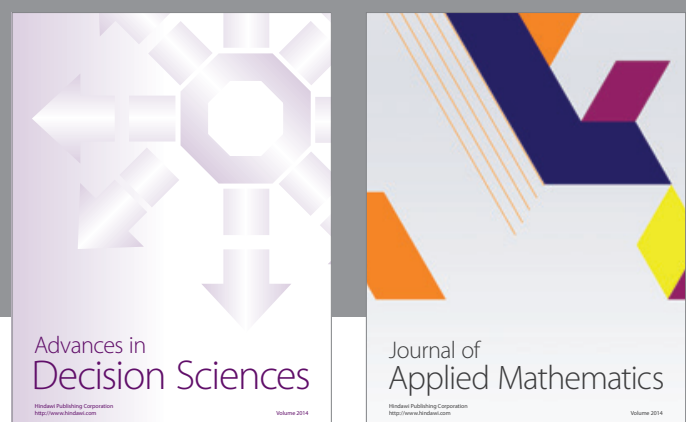

Journal of

Applied Mathematics
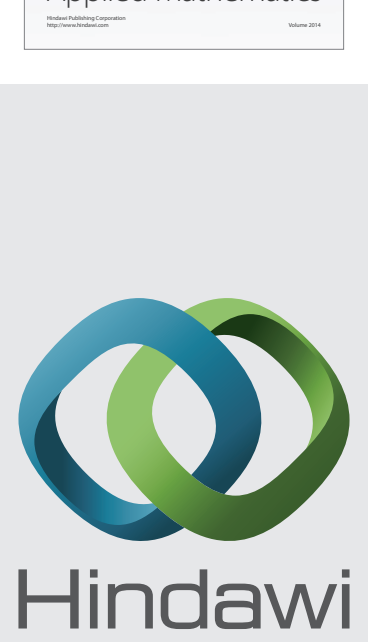

Submit your manuscripts at http://www.hindawi.com
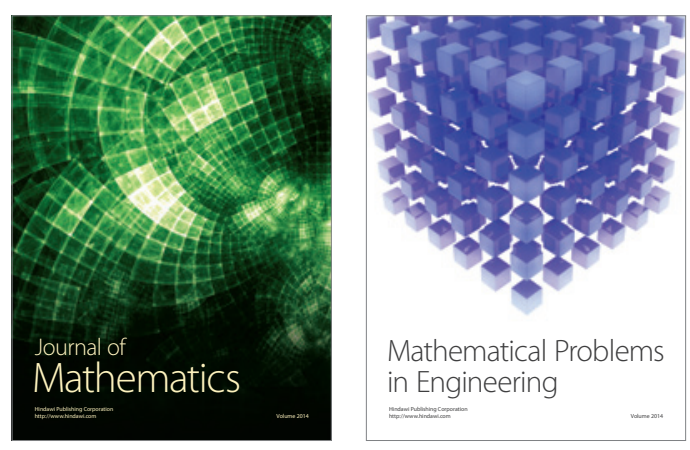

Mathematical Problems in Engineering
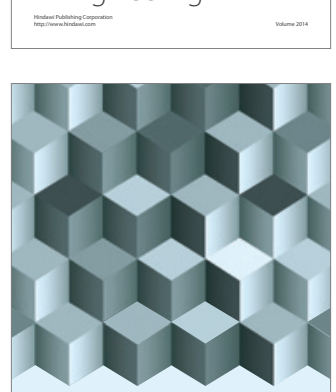

Journal of

Function Spaces
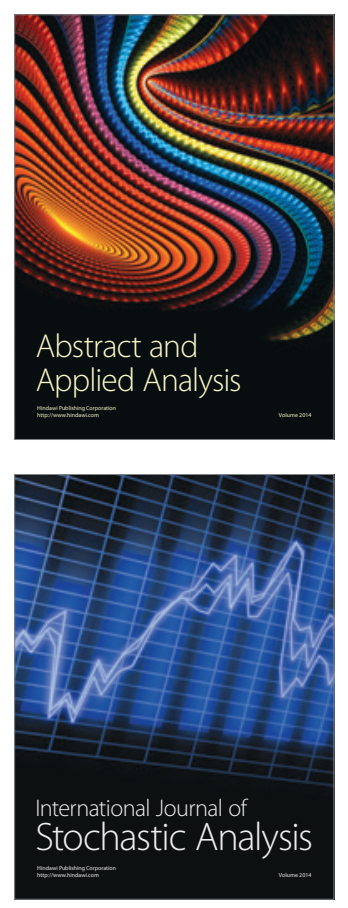

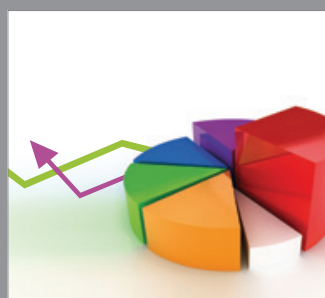

ournal of

Probability and Statistics

Promensencen
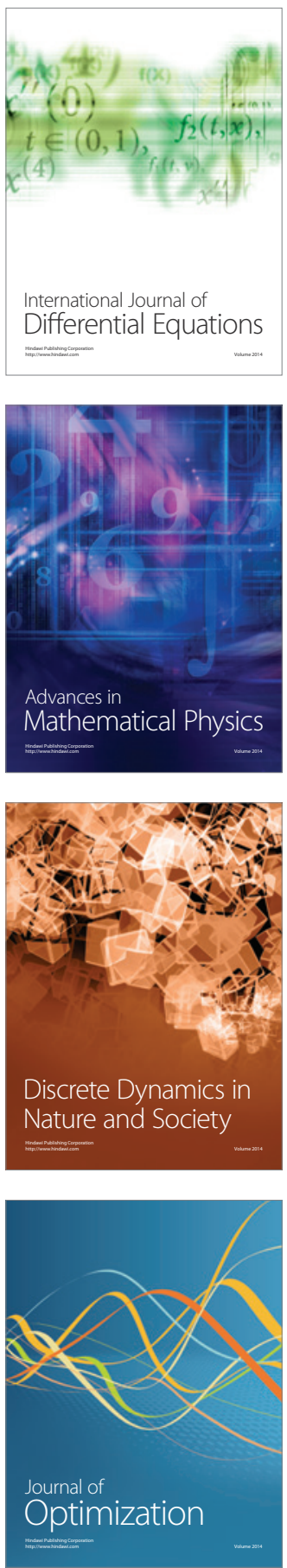